\title{
Micromagnetic Study of the Influence of Crystal Defects on Coercivity in Magnetite
}

\author{
BRUCE M. MOSKOWITZ \\ Department of Geology and Geophysics and Institute for Rock Magnetism, \\ University of Minnesota, Minneapolis
}

\begin{abstract}
A one-dimensional micromagnetic model is used to calculate the thermal dependence of microcoercivity $\left(h_{c}\right)$ produced by the unpinning of a domain wall (DW) from various types of defects in magnetite. Equilibrium solutions are found that minimize the magnetoelastic, anisotropy, exchange, magnetostatic, and external field energies with respect to the wall width $(w)$ and position of the wall relative to the defect. The defect may be a single dislocation, dislocation dipole, planar defect, or plauar defect bounded by two parallel dislocations. Wall pinning is produced by (1) microstress fields of dislocations, (2) local changes in exchange and anisotropy constants within a planar defect region, or (3) a combination of both effects. The calculations, using temperaturedependent parameters, predict the thermal dependence of $h_{c}(T)$ as a function of grain size, domain wall width, defect spacing, and type of defect. Results show thal, for grain sizes between 1 and $100 \mu \mathrm{m}, h_{c}(T)$ is usually a function of the wall width raised to some power $n$. The particular value of $\boldsymbol{n}$ is found to be a function of the DW-defect interaction spacing $(d / w)$, type of defect, and grain size. Also, within this size range, the wall width expands with temperature more gradually than classical theory predicts. The microcoercivity results are used with the theory of Xu and Merrill (1990) to predict the thermal dependence of the macroscopic coercivity $H_{c}$ in magnetite. For grains with low defect densilies, such as recrystallized magnetites, negative dislocation dipoles with $d / w \approx 0.1-1$ produce a thermal dependence of coercivity that agrees with experimental results. In the high defect density limit, a population of positive and negative dislocation dipoles with a distribution of dipole widths produce an $H_{c}(T)$ dependence consistent with experimental data from crushed and glass ceramic magnetites.
\end{abstract}

\section{INTRODUCTION}

Within the past few years there have been significant advances in our understanding of domain structure in small multidomain (MD) particles containing just a few domains [e.g., Dunlop, 1990]. Much of this new knowledge comes from micromagnetic calculations and domain observations. Yet, one of the fundamental outstanding problems in our understanding of the origin of magnetic stability and thermoremanence (TRM) in MD grains is the effect of stress and crystal defects on magnetic properties. It has long been recognized that crystal defects play an important role in determining hysteresis and susceptibility in MD particles [e.g., Kittel, 1949]. Defects can alter the magnetic domain structure or exert forces on domain walls (DW). For example, crystal defects are thought to be responsible for a variety of magnetic properties in $\mathrm{MD}$ particles of magnetite and titanomagnetite including the grain size dependence of coercivity $\left(H_{c}\right)$, remanence, and initial susceptibility [e.g., Stacey and Wise, 1967; Dunlop, 1986; Heider et al., 1087]; the temperature dependence of $H_{c}$ [e.g., Hodych, 1982; $X u$ and Merrill, 1989, 1992]; magnetic memory effect [e.g., Heider et al., 1992]; nucleation, denucleation, and pinning of domain walls during TRM acquisition [Halgedahl, 1991]; the unusual domain style in Ti-rich titanomagnetites [e.g., Halgedahl, 1987; Moskowitz et al., 1988]; and, the response of remanence to geological deformation [e.g., Borradaile, 1992]. However, the specific types and arrangements of defects and the exact mechanisms by which defects influence magnetization remain unclear.

Crystal defects can affect magnetization in two important ways. First, they can act as centers for the nucleation of new domain walls and aid magnetization reversal. This can occur at sites which

Copyright 1993 by the American Geophysical Union.

Paper number 93JB01719.

0148-0227/93/93JB-01719\$05.00 locally have large demagnetizing fields due to shape irregularities (e.g., sharp corners, microcracks), or at sites where the domain wall energy is locally changed by chemical or physical defects. Second, defects can pin existing domain walls and inhibit magnetization reversal by creating local energy barriers that impede wall movement. In the former case, defects decrease coercivity by creating nucleation sites, whereas in the latter case, defects increase coercivity by pinning domain walls.

There are many different types of crystal defects that can interact with domain walls including, point defects, dislocations, stacking faults, subgrain boundaries, inclusions, and voids. Defects may also interact with one another. For example, point defects such as vacancies can coalesce to form dislocation loops and single dislocations can disassociate into partial dislocations and stacking faults or associate to form complex arrays [e.g., Hull, 1975]. Because of the variety of different types and arrangements of defects and their interactions with one another and with domain walls, the effects of defects on magnetic behavior can be complex [e.g., Träuble, 1969; Xu and Merrill, 1989]. For example, the microstress fields of dislocations may trap remnants of domain walls after application of a large saturating field and provide sites for "renucleation" after removal of the field [e.g., Halgedahl, 1991]. Other defects, such as extended planar defects, may produce a local change in magnetic anisotropy and exchange energies, making them favorable sites for domain nucleation or wall pinning.

Several simplifying assumptions can be made to render the theoretical treatment tractable. Of the many different types of defects, dislocations are considered to be the most common. Pinning of domain walls by dislocations is modeled as the magnetoelastic interaction between domain walls and the stress fields of the dislocations. This is the approach taken by $X_{u}$ and Merrill $[1989,1990 a, 1992]$ in their theoretical analysis of microcoercivity $\left(h_{c}\right)$ and macroscopic or bulk coercivity $\left(H_{c}\right)$ in MD inagnetite. Microcoercivity, $h_{c}$, is the field necessary to unpin a single wall 
from a defect and is related to the maximum pinning force of the defect. Usually, it is not the same as $H_{c}$ measured from a hysteresis loop [ $X u$ and Merrill, 1989]. The microcoercivily is related to the bulk coercivity, sometimes in a nonlinear fashion, by statistically averaging over many defects, walls, and grains [Xu and Merrill, 1990a].

Despite the theoretical complexities involved in wall-defect interactions, experimental data for magnetite suggest a simple result. Hodych [1982, 1986] showed that the temperature dependence of $H_{c}(\mathrm{~T})$ between 120 and $300 \mathrm{~K}$ was proportional to $\lambda / M_{s}$ for various samples of MD magnetite, where $\lambda$ is the magnetostriction constant and $M_{s}$ is the saturation magnetization. This result suggested that $H_{c}$ was predominantly controlled by stress, presumably by microstress fields of defects. Later it was found that the $\lambda / M_{s}$ dependence of $H_{c}$ was valid up to the Curie temperature of magnetite [Xu and Merrill, 1992]. However, $X u$ and Merrill $[1989,1990 a]$ found these results surprising because there was no explicit dependence on the wall width, which should vary with temperature, or on the demagnetizing factor, which can be significant in MD grains. The latter is particularly important when considering the potentially large demagnetizing fields associated with denucleated "SD states" observed by Halgedahl [1991]. Most theories of defect-controlled microcoercivity predict that $h_{c}(T)$ $\propto w(T)^{n}$, where $w$ is the wall width and $n$ is a constant that can be less than or greater than zero depending on the type or arrangement of defects [e.g., Träuble, 1969; Xu and Merrill, 1989]. According to classical domain theory [Landau and Lifshitz, 1935], the wall width in an infinite material is $w \propto\left(A / K_{l}\right)^{1 / 2}$, where $A$ is the exchange constant and $K$, is the magnetocrystalline anisotropy constant. In magnetite, this type of wall will expand rapidly with increasing temperature owing to the rapid decrease in $K_{l}(T)$ at elevated temperature. To resolve the discrepancy between theory and experiment, $X u$ and Merrill [1992] assumed that the variation of wall width with temperature in magnetite is mainly determined by stress and magnetostatic energy instead of magnetocrystalline anisotropy. Using an Amar-type calculation, $\mathrm{X} u$ and Merrill [1990b] show that this assumption results in a gradual expansion of the wall with temperature; hence, $H_{c}(T) \approx \lambda / M_{s}$. Nevertheless, an exceedingly large magnitude of residual stress was necessary to reconcile theory with observations [ $X u$ and Merrill, 1990b]

In this paper, a different approach is used to study the effects of defects on coercivity. We consider a theoretical model of wall pinning by a defect in magnetite within the framework of micromagnetism in the spirit of recent domain calculations [e.g., Moon and Merrill, 1984; Newell et al., 1990]. Instead of simply equating the maximum pinning force of a dislocation with $h_{c}$ [e.g., Träuble 1969; Xu and Merrill, 1989, 1990a, 1992], we have taken the domain wall energy to be the sum of magnetoelastic, anisotropy, exchange, magnetostatic, and external field energies and have

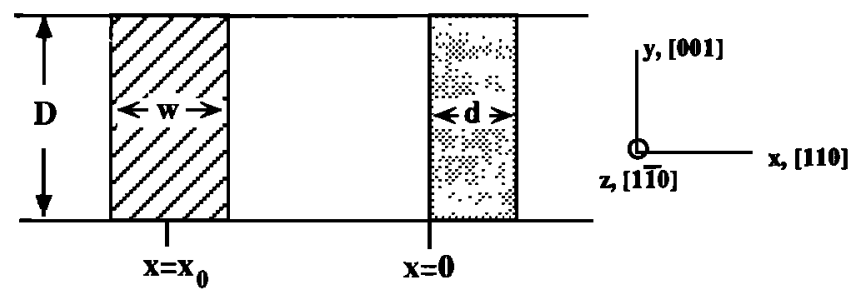

Fig. 1. Model geometry and wall coordinate system. The plate thickness (i.e., grain size) is $D$, wall width is $w$, and defect width is $d$. The defect is located at $x=0$, and the center of the wall is located at $x=x_{0}$. The wall coordinate system is for a $180^{\circ}$ (110) wall interacting with a screw dislocation or planar defect. found solutions that minimize this energy with respect to the wall width and position of the wall relative to the defect. An advantage of this formulation is that the temperature dependence for the wall width is calculated directly. By necessity, the present model, like former ones dealing with defects, is one dimensional and assumes a particular magnetization distribution a priori. A complete threedimensional solution is not yet possible. Hilzinger [1977] and Aharoni [1985] have formulated micromagnetic models to describe the pinning of a wall at a planar defect. Our model follows closely the formulation of Aharoni [1985] but is extended to model different types of defects and to predict how the thermal dependence of coercivity varies with the type of defect.

\section{Model Geometry}

\section{MICROMAGNETIC MODEL}

Figure 1 shows the geometry of the model, which consists of a semi-infinite plate of thickness $D$, infinite in the $x$ and $z$ directions and extending from $-D / 2$ to $+D / 2$ in the $y$ direction. The coordinate system shown in Figure 1 is similar to the one used by $X u$ and Merrill [1989] and is appropriate for magnetite with easy axis of magnetization along $<111>$. In this case, a planar $180^{\circ} \mathrm{DW}$ of width $w$ in the (110) plane separates two domains magnetized along the $[1 \overline{1} 1]$ and $[\overline{1} 1 \overline{1}]$ easy directions. The $x$ axis is normal to the wall plane along the [110] direction, the $y$ axis is perpendicular to the dislocation line along the [001] direction, and the $z$ axis is within the wall plane. parallel to the dislocation line, along the $[1 \overline{1} 0]$ direction. Magnetization rotates within the $y-z$ plane and the wall moves along the $x$ direction. A "wall" coordinate system is used because it simplifies the calculation of magnetoelastic energy by eliminating interactions when the dislocation line or its Burgers vector are not within the wall plane [Träuble, 1969; Xu and Merrill, 1989]. The DW interacts with a defect region of width $d$. It is assumed that the DW and defect are infinitely extended and parallel to each other. The defect may be a single dislocation $(d=0)$, two dislocations (dislocation dipole), a planar defect, or a planar defect bounded by two dislocations. A planar defect is characterized by a local variation in exchange coupling and crystalline anisotropy within the defect. Although details of this local perturbation are not known, it is approximated by an exchange $\left(A^{\prime}\right)$ and anisotropy $\left(K^{\prime}\right)$ constant that are different within the defect region $(0<x<d)$ than in the rest of the plate $(x<0$ and $x>d$ ) [Aharoni, 1985].

\section{Total Wall Energy}

The total free energy of a domain wall $E_{t}$ interacting with a dislocation, dislocation dipole, or planar defect, is the sum of several energy terms

$$
E_{s}\left(x_{0}, w\right)=E_{s}+E_{a}+E_{c}+E_{m}+E_{h}
$$

where $x_{0}$ is the position of the center of the wall, $E_{s}$ is the magnetoelastic interaction energy with a particular type of defect, $E_{a}$ is the anisotropy energy, $E_{e}$ is the exchange energy, $E_{m}$ is the magnetostatic energy, and $E_{h}$ is the external field energy. The total energy is a function of two variables, the position of the DW relative to the defect and the width of the DW. The functional forms for the anisotropy, exchange, magnetostatic, field, and magnetoelastic energies are adapted from Aharoni [1985] and $X u$ and Merrill [1989]. Ideally, to solve the wall-pinning problem represented by (1), $E_{1}$ is minimized to give the equilibrium magnetization distribution. However, this condition is insoluble analytically; to circumvent this difficulty an analytical approxi- 
mation to the direction cosines of the magnetization vector in the plate is chosen a priori and only the width of the wall and its location are allowed to vary.

\section{Wall Siructure}

A rigid, planar Bloch DW is centered at $x=x_{0}$. The magnetization within the wall corresponds to the Dietze-Thomas model [Dietze and Thomas, 1961; Aharoni. 1985]. For this wall model, the magnetization distribution is given by

$$
\sin \varphi(x)=\frac{w^{2}}{w^{2}+\left(x-x_{0}\right)^{2}}
$$

where $\varphi(x)$ is the angle between the magnetization and the easy axis and $w$ is proportional to the wall width. A uniaxial anisotropy is assumed. The Dietze-Thomas wall model is sufficiently close to the exact solution of the Landau-Lifshitz model [Jakubovics, 1978; Aharoni, 1985] but has the advantage that the direction cosines given by (2) are rational algebraic functions of $x$, making the various energy functionals simpler to integrate and avoiding numerical integrations.

In the wall-coordinate system shown in Figure 1 , the DW magnetic structure is described by the direction cosines, $\alpha_{i}$ 's, given by

$$
\alpha_{x}=0, \quad \alpha_{y}=-\cos \left(\varphi(x)-\varphi_{0}\right), \quad \alpha_{z}=\sin \left(\varphi(x)-\varphi_{0}\right)
$$

where $\varphi(x)$ is given by (2) and $\varphi_{0}=\cos ^{-1}(1 / \sqrt{3})$ is the angle between the $y$ axis and the easy axis of magnetization $[X u$ and Merrill, 1989]. It is also assumed that the wall structure does not change its form at elevated temperatures approaching the Curie temperature.

\section{Magnetoelastic Interaction Energy}

The magnetoelastic interaction energy for a dislocation follows the derivation of $X u$ and Merrill [1989]. In a cubic crystal like magnetite, the magnetoelastic interaction energy is given by

$$
\begin{aligned}
E_{s}= & -\frac{3}{2} \lambda_{100} \int\left(\alpha_{1}^{2} \sigma_{11}+\alpha_{2}^{2} \sigma_{22}+\alpha_{3}^{2} \sigma_{33}\right) d V \\
& -3 \lambda_{111} \int\left(\alpha_{1} \alpha_{2} \sigma_{12}+\alpha_{2} \alpha_{3} \sigma_{23}+\alpha_{1} \alpha_{3} \sigma_{13}\right) d V
\end{aligned}
$$

where $\sigma_{i j}$ are the stress components of the dislocation, $\alpha_{i}$ are the direction cosines of magnetization, and $\lambda_{I I I}$ and $\lambda_{100}$ are the magnetostriction constants. The $\sigma_{v}$ and $\alpha_{t}$ are taken with respect to the cube axes. We consider a straight screw dislocation lying parallel to the wall in the $z$ direction. The effects of an edge dislocation are similar to those of a screw dislocation, but calculations are easier for the latter case [Xu and Merrill, 1989].

Under the approximation that $D \gg>w$, the variation of stress within the slab becomes a step function centered on the dislocation at $x=0[X u$ and Merrill, 1989]. For this case, the only non zero stress component of a screw dislocation, averaged over the plane of the wall, is

$$
\bar{\sigma}_{y z}(x)=\frac{b \mu}{2 D} \operatorname{sign}(x)
$$

In (5) $b$ is the magnitude of the Burgers vector, $\mu$ is the shear modulus, sign $(x)=1$ for $x \geq 0$ and $\operatorname{sign}(x)=-1$ for $x<0$, and the $y z$ subscripts refer to the wall coordinates. For magnetite, the stepfunction approximation is valid for $D>1 \mu \mathrm{m}[X u$ and Merrill, 1989] and thus places a lower limit on $D$ used in the models. After transforming (4) into wall coordinates, the magnetoelastic energy per unit wall area for a $(110) 180^{\circ}$ wall subjected to a stress field given by (5) is

$$
E_{s}=-3 \lambda_{111} \int_{-\infty}^{\infty} \bar{\sigma}_{y z}(x) \alpha_{y} \alpha_{z} d x
$$

Substituting (3) and (5) into (6) and integrating gives

$$
E_{s}=\frac{\lambda_{111} \mu b w}{2 D}\left[2 \sqrt{2} C\left(\frac{x_{0}}{w}\right)-B\left(\frac{x_{0}}{w}\right)\right]
$$

with

$$
\begin{gathered}
C(x)=\frac{x}{1+x^{2}}+\tan ^{-1}(x) \\
B(x)=\frac{\sqrt{2+x^{2}}}{1+x^{2}}+\log \frac{1+\sqrt{2+x^{2}}}{\sqrt{1+x^{2}}}
\end{gathered}
$$

Now consider two screw dislocations at $x=0$ and $x=d$ whose Burgers vectors are parallel but can have the same or opposite signs. This configuration is called a dislocation dipole and is characterized by its width $d$ and sign [e.g., Hull, 1975]. A positive (negative) dipole occurs when the Burgers vectors have the same (opposite) sign. Theoretically, only edge dislocations can form stable dipole configurations; however, stable screw dipoles have been observed in nickel ferrite spinels [Veyssière et al., 1978], and it will be assumed that they can form in magnetite as well. Following the derivation by $X u$ and Merrill [1989], the stress for a dipole is

$$
\bar{\sigma}_{y x}(x)=\frac{b \mu}{2 D}[\operatorname{sign}(x) \pm \operatorname{sign}(x-d)]
$$

where the plus and minus signs correspond to a positive or negative dipole, respectively. Using (9) with (6), the magnetoelastic interaction energy for a \pm dipole and (110) $180^{\circ}$ wall is

$$
\begin{aligned}
E_{s} & =\frac{\lambda_{111} \mu b w}{2 D}\left\{\left[2 \sqrt{2} C\left(\frac{x_{0}}{w}\right)-B\left(\frac{x_{0}}{w}\right)\right]\right. \\
& \left.\mp\left[2 \sqrt{2} C\left(\frac{d-x_{0}}{w}\right)+B\left(\frac{d-x_{0}}{w}\right)\right]\right\}
\end{aligned}
$$

When $d / w>>1$, the dipole pair is very far apart and (10) reduces to (7) appropriate for a wall pinned by a single dislocation. When $d / w<<$, the stress fields of the two dislocations of a negative dipole will cancel one another and the dipole will appear to be annihilated $\left(E_{s}=0\right)$, and no wall pinning will occur. For a positive dipole, the stress fields of the two dislocations add and wall pinning is the same as a single dislocation but with twice the magnitude. The width of the dipole is assumed to be fixed and independent of temperature, meaning that the model does not take into account any thermal migration of dislocations such as glide or climb.

The one dimensionality of the present model implies the rigidwall approximation, which means that the microstress fields of dislocations do not affect the Bloch wall structure in any significant way. This is a first-order approximation because domain walls may be flexible and bow out between pinning centers under the influence of a nonuniform stress field [e.g., $X u$ and Merrill, 1989, 1992]. The conditions under which DW bowing will be important in magnetite are difficult to estimate. However, our micro- 
magnetic model is restricted to the interaction of a single DW and defect, which are both infinite in extent and parallel to each other. In this case, the microstress field of the dislocation is uniform over the entire area of the wall and DW bowing can be neglected.

\section{Anisotropy Energy}

To calculate the anisotropy energy, we use the standard assumption of uniaxial anisotropy but consider both magnetocrystalline and magnetostrictive contributions. The magnetostrictive term is associated with magnetostriction stresses in the domains and is not related to microstress fie]ds of defects [e.g.. $X u$ and Merrill, 1990b; Moon, 1991]. For a (110)180 wall, the anisotropy energy per unit wall area is

$$
E_{\alpha}=K_{u} \int_{-\infty}^{\infty} \sin \varphi^{2}(x) d x
$$

with

$$
K_{u}=\frac{2}{3}\left|K_{1}\right|+\frac{9}{2} \mu \lambda_{111}^{2}
$$

where $K_{1}$ is the magnetocrystalline anisotropy constant.

Following Aharoni [1985], for a planar defect of width $d$ located between $0<x<d$ and anisotropy constant $K_{u}^{\prime}$ different from its value outside the defect, (11) integrates to

$$
E_{a}=\frac{K_{u} w}{2}\left\{\pi+\left(\frac{K_{u}^{\prime}}{K_{u}}-1\right)\left[C\left(\frac{d-x_{0}}{w}\right)+C\left(\frac{x_{0}}{w}\right)\right]\right\}
$$

where $C(x)$ is given in (8). If $K_{u}{ }^{\prime}=K_{u}$, the anisotropy energy is independent of the wall position and will not contribute to wall pinning.

\section{Exchange Energy}

In the framework of micromagnetism, the exchange interactions are treated in a classical fashion by allowing neighboring spins to vary in a continuous fashion with position [Kittel, 1949]. Under this assumption, using (2) and transforming to wall coordinates the exchange energy per unit wall area is given by

$$
E_{.}=A \int_{-\infty}^{\infty}\left(\frac{\partial \varphi(x)}{\partial x}\right)^{2} d x
$$

where $A$ is the exchange constant.

For a planar defect of width $d$ and anisotropy constant $A$ different from its value outside the defect, (14) integrates to

$$
E_{e}=\frac{2 A}{w}\left\{\pi(\sqrt{2}-1)+\left[\frac{A^{\prime}}{A}-1\right]\left[E\left(\frac{d-x_{0}}{w}\right)+E\left(\frac{x_{0}}{w}\right)\right]\right\}
$$

with

$$
E(x)=\frac{x}{1+x^{2}}-\tan ^{-1}(x)+\sqrt{2} \tan ^{-1}\left(\frac{x}{\sqrt{2}}\right)
$$

Like the anisotropy term, exchange can pin the wall at the planar defect only when $A^{\prime}$ does not equal $A$ [Aharoni, 1985].

\section{Magnetostatic Energy}

The magnetostatic energy per unit wall area for the DietzeThomas wall in a semi-infinite plate has a simple analytical solution [Dietze and Thomas, 1961; Aharoni, 1985] given by

$$
E_{m}=\frac{2 \pi^{2} \mu_{0} M_{s}^{2} w^{2}}{D} \log \left(1+\frac{D}{2 w}\right)
$$

where $M_{s}$ is the saturation magnetization and $\mu_{0}$ is the permeability of free space. For one wall, (17) shows that $E_{m}$ is independent of wall position but is a function of the wall width and plate thickness. For more than one wall, the magnetostatic energy of the domains as well as the walls must be determined and no longer has a simple analytic solution. Note that when $D$ becomes very large, $E_{m}$ approaches zero.

\section{Extemal Field Energy}

An external field $H_{0}$ applied along the easy direction will move the wall along the positive $x$ direction. If the wall is moved a small distance by $H_{0}$, the external field energy per unit wall area [Aharoni, 1985] is

$$
E_{h}=-2 \mu_{0} H_{0} M_{s} x_{0}
$$

Equation (18) was derived under the assumption that the wall is far away from any crystal surface or any other wall.

\section{Energy Minimization Procedure}

For a wall interacting with a defect, the total free energy $E_{1}$ of the wall is given by the sum of (10), (13), (15), (17), and (18). $\mathrm{E}_{\mathrm{l}}$ is a function of two independent variables, wall position $x_{0}$ and wall width $w$, and several model parameters including material constants, temperature, and external field. An equilibrium configuration is determined by equating the partial derivatives of $E$ with respect to $x_{0}$ and $w$ to zero

$$
\frac{\partial E_{t}}{\partial x_{0}}=0, \frac{\partial E_{t}}{\partial w}=0
$$

Equation (19) gives a relationship between $x_{0}, w$, and $H_{0}$ and is a set of two nonlinear equations in two unknowns that can be easily solved because all the functional fonns have analytical solutions [Aharoni, 1985]. The complete form of (19) is given in the appendix. The nonlinear equations (19) are solved numerically using a variable metric algorithm [Press et al., 1988], which finds a stable solution by requiring the second-derivative matrix to be positive definite.

To calculate $h_{c}$, solutions to (19) are obtained for a given value of $H_{\theta}$. This process is continued as $H_{0}$ is increased. The solutions for $x_{0}$ increase with increasing $H_{0}$ until some value $H_{\max }$ is reached, at which point there are no longer any stable minimum solutions to (19). Therefore, when $H_{0}=H_{\max }$, the wall breaks away from the defect and jumps to infinity along the positive $x$ direction [Aharoni, 1985]; the microcoercivity is then defined as $h_{c}=H_{\text {max }}$.

Microcoercivity was determined as a function of temperature, plate thickness (i.e., grain size), defect width, and type of defect. A useful dimensionless parameter for describing the results is the reduced defect width $d / w_{0}$, where $w_{0}=\left(A_{0} / K_{0}\right)^{1 / 2}$ and is a measure of the wall width at room temperature. This parameter sets the size of the defect width $(d)$, which remains constant with temperature. However, $d / w(T)$ decreases with increasing temperature 
because of the expansion of the wall widh with temperature. The material constants used in the model are those appropriate for magnetite and are listed in Table 1.

\section{RESULTS}

\section{Thermal Dependence of Wall Width}

An advantage of the present micromagnetic formulation is that the thermal dependence of the wall width is determined directly from the energy minimization procedure. Model calculations of the thermal dependence of the wall width for dislocation dipoles $\left(d / w_{0}=0.1\right)$ with grain sizes $(D)$ of $1,10,100$, and $1000 \mu \mathrm{m}$ are shown in Figure 2. Also included in Figure 2 are three estimates of the classical thermal dependence of wall width, each of which arises from one of the following three types of anisotropy: (1) magnetocrystalline/magnetoelastic anisotropy [e.g., $X u$ and Merrill. $1990 b$ ], for which

$$
K=\frac{2}{3}\left|K_{1}(T)\right|+\frac{9}{2} \mu \lambda_{111}(T)^{2}
$$

(2) stress anisotropy [Moskowitz and Halgedahl, 1987; Xu and Merrill, 1990b], for which

$$
K=\frac{2}{3}\left|K_{1}(T)\right|+\frac{3}{2} \sigma \lambda_{111}
$$

where $\sigma$ is a stress sufficient to make the second term of (21) equal the magnetocrystalline anisotropy at room temperature; and (3) shape anisotropy, for which

$$
K=\frac{2}{3}\left|K_{1}(T)\right|+2 \pi M_{s}(T)^{2}
$$

Model results fall between the two extreme classical estimates of (20) and (22). This result is readily explained by the observation that for smaller grain sizes, the magnetostatic energy of the wall outweighs the uniaxial anisotropy term, and the wall width expands slowly with temperature, approximately as (22). For larger grain sizes, in contrast, the magnetostatic energy of the wall becomes less important and the wall width expands more rapidly with temperature and approaches the classical limit given by (20). Results for the thermal dependence of $w(T)$ for $D=10 \mu \mathrm{m}$ are approximately the same as the stress model used by $X u$ and Merrill $[1990 b]$ in their Amar-type calculations. For magnetite with a grain size less than $\approx 50 \mu \mathrm{m}$, the model calculations predict a gradual expansion of the wall width with temperature instead of the rapid expansion predicted by (20).

Unlike the effects of grain size, there is a negligible effect on $w(T)$ due to variations in $d / w_{0}$ or type of defect because the model does not take into account any direct influence of the dislocation stress field on the wall structure. Only the absolute magnitude of $w$ varies slightly with these model conditions.

\section{Dislocation Models}

The first class of models investigated is one where a DW interacts with a dislocation dipole. Typical results for the thermal dependence of the microcoercivity in a $10-\mu \mathrm{m}$ grain containing negative dislocation dipoles with $d / w_{0}=0.1,1$, and 10 are shown in Figure 3. The solid lines correspond to $\left(\lambda / M_{s}\right) w,\left(\lambda / M_{s}\right) w^{1 / 2}$, and $\lambda / M_{s}$ for $d / w_{\sigma}=0.1,1.0$, and 10 , respectively. The results for all other $d / w_{0}$ values fall within the curves calculated for 0.1 and 10. The model for $d / w_{0}=10$ gives a result that is identical with the $h_{c}(T)$ dependence due to a single dislocation [e.g., Stacey and Wise, 1967; $X u$ and Merrill, 1989]. Here, the dislocations are far enough apart relative to the wall width that the wall senses only one dislocation and $h_{c}(T)$ is independent of the wall width.

The microcoercivity for a wall interacting with a negative or positive dislocation dipole can be conveniently systematized in terms of the wall width exponent $n$, namely, $h_{c}(T) \propto\left(\lambda / M_{s}\right) w(T)^{n}$. The wall width exponent is determined by linear least squares fitting of the model results; a good fit can usually be obtained. The exponent $\mathrm{n}$ as a function of $d / w_{0}$ for constant $D$, or $D$ for constant $d w_{0}$, for positive and negative dipoles is shown in Figure 4. Negative dipoles result in a microcoercivity that is inversely proportional to the wall width with $n$ varying from -1 to 0 as $d / w_{0}$ varies from 0.1 to 10 , respectively. In contrast, positive dipoles result in a microcoercivity that is proportional to the wall width with $n$ reaching a maximum value of $\approx 0.6$ near $d / w_{0}=1$ and decreasing to zero as $d / w_{b}$ approaches 0.1 and 10 . Considering both positive and negative dipoles, $n$ may be expected to vary between -1 and 0.6 and produce $h_{c}(T)$ 's that may vary either slower or faster with temperature than $\lambda / M_{s}$.

The contrasting behavior produced by positive and negative dipoles is related to the different stress distributions of the two types of dipoles. For negative dipoles, stress is a maximum between the dislocations $(0<x<d)$ and zero outside $(x<0, x>d)$. As the wall width increases relative to the width of the dipole, the stress field of the negative dipole becomes less effective at pinning the DW. Hence, one would predict $h_{c}(T)$ to vary inversely with $w(T)$ over a limited range of $d / w_{0}$. For positive dipoles, the stress distribution is the opposite of that for a negative dipole. Stress is zero between the dislocations of a positive dipole and a maximum outside the dipole. As the width of the DW increases, the DW will increasingly interact with more of the stress field outside the dipole. Therefore, one would predict $h_{c}(T)$ to vary positively with $w(T)$ over a limited range of $d / w_{0}$. For large MD grains $(D>100$

TABLE 1. Material Constants for Magnetite

\begin{tabular}{cccl}
\hline Material Constant & $\begin{array}{c}\text { Room Temperature } \\
\text { Value }\end{array}$ & $\begin{array}{c}\text { Thermal } \\
\text { Dependence }\end{array}$ & \multicolumn{1}{c}{ Reference } \\
\hline$M_{s}$ & $480 \mathrm{kA} \mathrm{m}^{-1}$ & $\left(\mathrm{~T}_{c}-\mathrm{T}\right)^{04}$ & Pauthenet [1952] \\
$K_{l}$ & $-1.35 \times 10^{4} \mathrm{~J} \mathrm{~m}^{-3}$ & $\left(\mathrm{~T}_{c}-\mathrm{T}\right)^{31}$ & Fletcher and O'Reilly [1974] \\
$\lambda_{I I}$ & $78 \times 10^{-6}$ & $\left(\mathrm{~T}_{c}-\mathrm{T}\right)^{0.9}$ & Moskowitz [1993] \\
$A$ & $1.29 \times 10^{-11} \mathrm{~J} \mathrm{~m}^{-1}$ & $\mathrm{M}_{c}^{1.8}$ & Heider and Williams [1988] \\
$\mu$ & $9.6 \times 10^{10} \mathrm{~N} \mathrm{~m}^{-2}$ & constant & Stacey and Wise [1967] \\
$b$ & $0.84 / \sqrt{2} \mathrm{~nm}^{1.9}$ & constant & Stacey and Wise [1967] \\
$T_{c}$ & $575^{\circ} \mathrm{C}$ & constant & \\
\hline
\end{tabular}

$M_{s}$, saturation magnetization; $K_{l}$, magnetocrystalline anisotropy constant; $\lambda_{, \prime \prime}$, magnetostriction constant; $A$, exchange constant; $\mu$, elastic shear modulus; $b$, magnitude of the Burgers vector; $T_{c}$, Curie Temperature. 


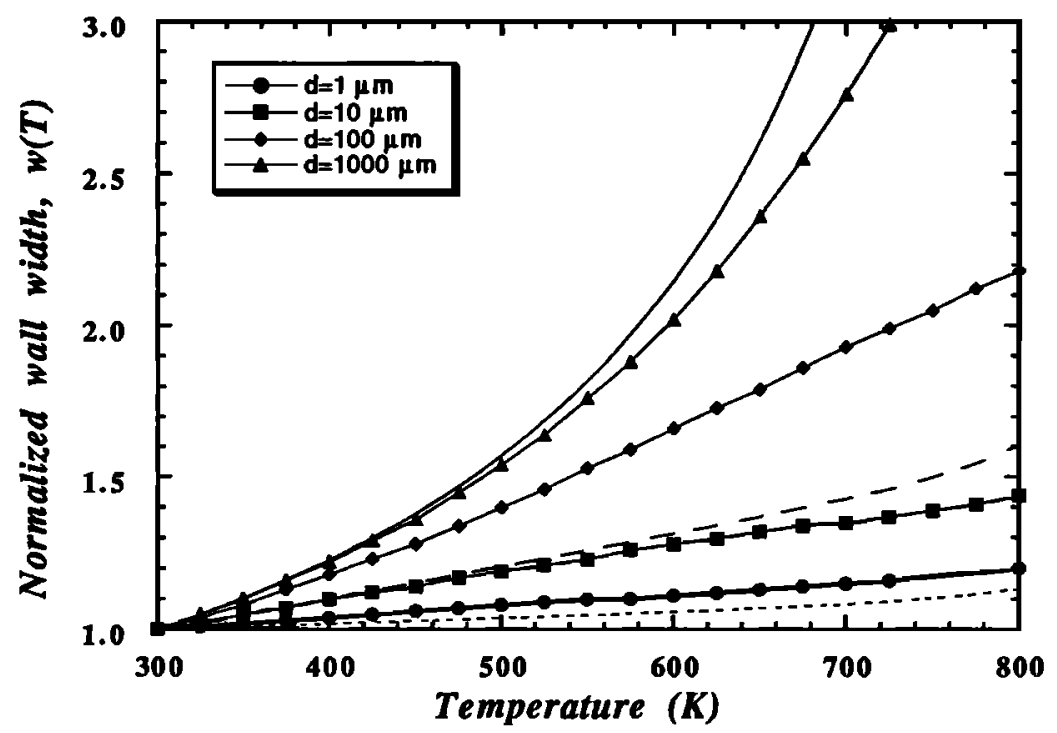

Fig. 2. The temperature dependence of normalized domain wall width $w(T) / w_{o}$ produced by a negative dislocation dipole $\left(d / w_{o}=\right.$ $0.10)$ with grain sizes $(D)$ of 1 (solid circles), 10 (solid squares), 100 (solid diamonds), and 1000 (solid triangles) $\mu \mathrm{m}$. Classical estimates of the thermal expansion of the wall width in bulk material are shown for (1) magnetocrystalline/ magnetoelastic anisotropy (solid line), (2) stress anjsotropy (long dashed line), and (3) shape anisotropy (short dashed line).

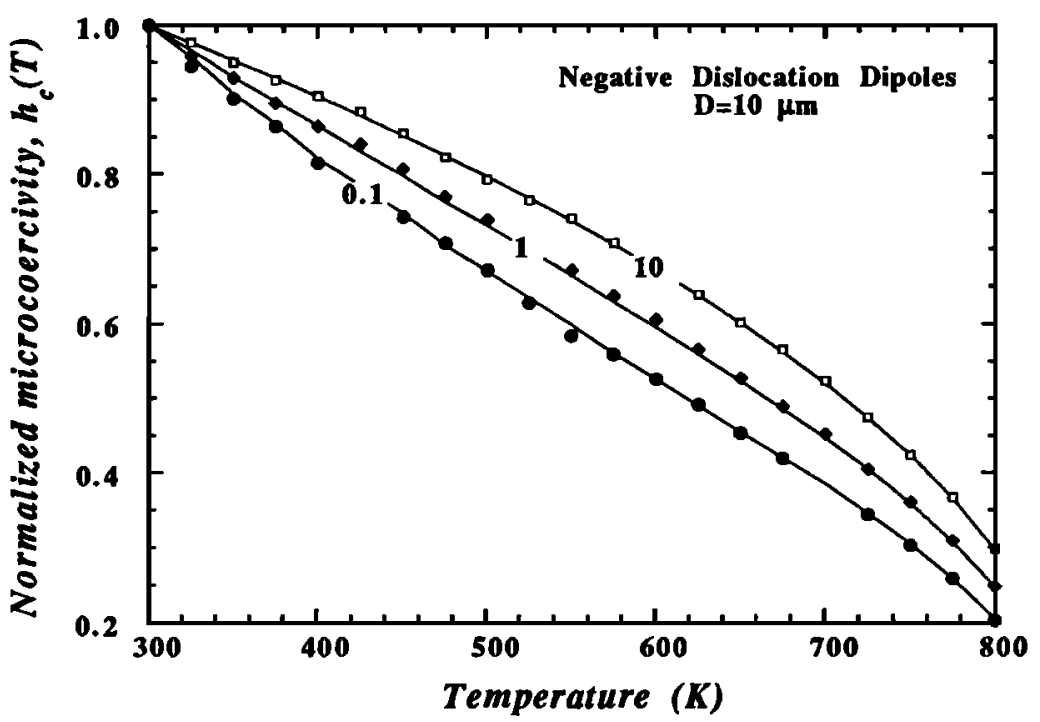

Fig. 3. Normalized thermal dependence of microcoercivity produced by negative dislocation dipoles with $d / w_{0}=0.1$ (solid circles), 1 (solid diamonds), and 10 (solid squares). The solid lines correspond to the thermal dependence of $(\lambda / M,) w^{-1},(\lambda / M,) w^{\text {th }}$, and $\lambda M_{1}$ for $d / w_{0}=0.1,1$, and 10 , respectively. Gran size is $10 \mu \mathrm{m}$.

$\mu \mathrm{m})$, the wall width increases more rapidly with temperature than $\lambda / M$, decreases and therefore leads to an initial increase in $h_{c}(T)$ with temperature (Figure 5). Only at low temperatures $\left(\mathrm{T}<200^{\circ} \mathrm{C}\right.$ ) in large MD grains can $h_{c}(T)$ be approximated by a simple power law in the wall width for which $n \approx 0.6$.

\section{Planar Defect Models}

Planar defects are defined as an area of material of width $d$ in which the exchange $\left(A^{\prime}\right)$ and anisotropy $\left(K_{u}\right)$ constants change abruptly by a fixed percentage increase or decrease from their values in the bulk material. The temperature dependence of the constants is assumed to be the same inside and outside the defect. An increase (decrease) in the constants causes the defect to be a region of high (low) wall energy. The former is termed a "strong" defect and the latter a "weak" defect. Demagnetizing effects resulting from the formation of magnetic poles or spike domains at the boundary are neglected [e.g., Cullity, 1972], a reasonable assumption for small defects $\left(d / w_{0}<1\right)$. It is assumed also that there is no strain associated with the planar defect. A change in the exchange or anisotropy constant in the planar defect could be accomplished through changes in bond lengths or angles between magnetic ions inside and outside the defect. Exactly how this occurs or the magnitude of the effect is uncertain, but domain walls have been observed to be pinned at planar defects in magnetite [Jakubovics et al., 1978]. The planar model may represent a stacking fault, antiphase domain boundary, or a second phase. In the latter case, a nonmagnetic phase, such as oxy-exsolution lamellae of ilmenite. can be represented as a region where $A^{\circ}$ and $K_{u}{ }^{\prime}$ are zero.

Several different planar defect models were studied. The temper- 

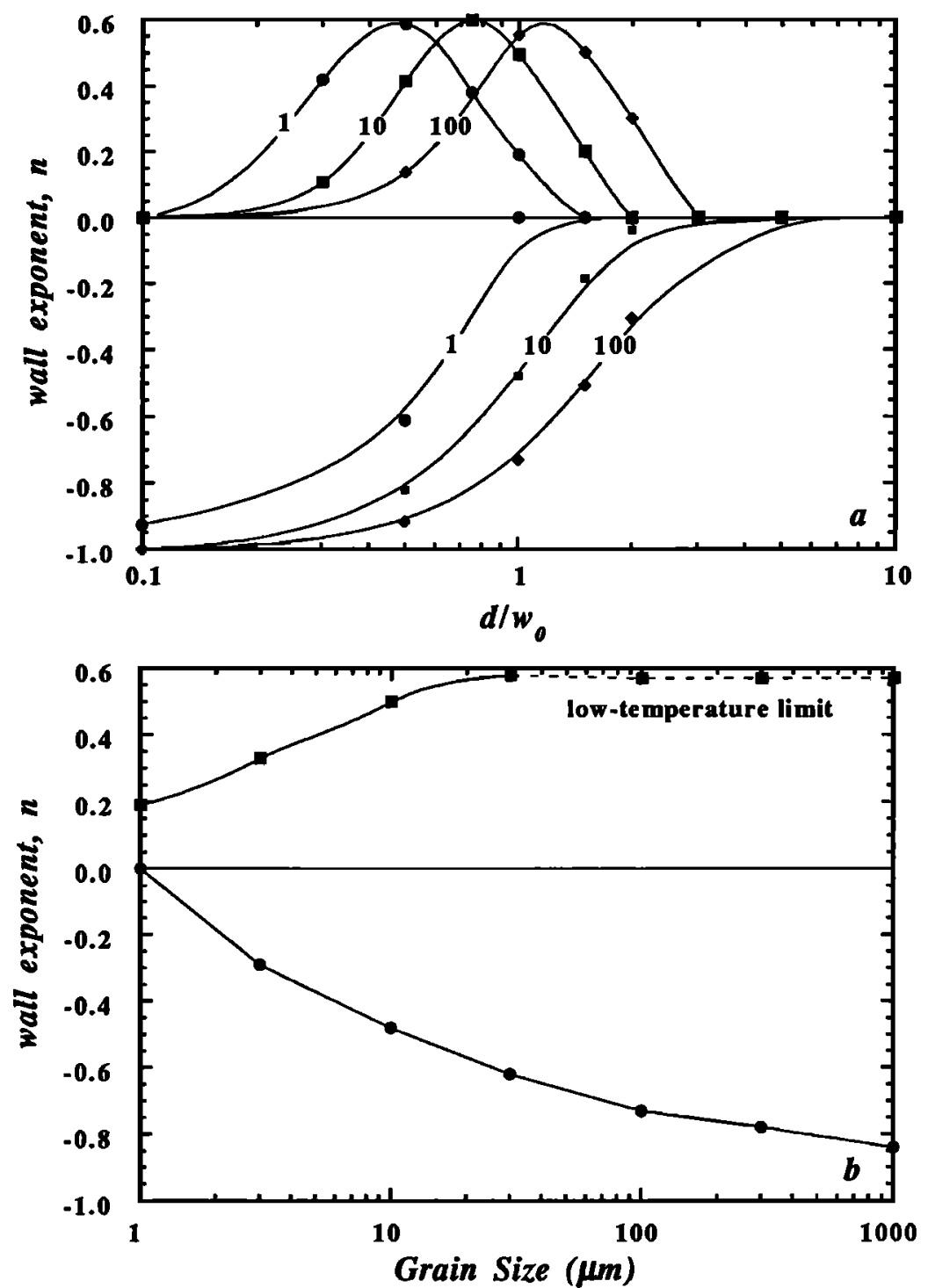

Fig. 4. (a) Wall exponent $n$ as a function of $d / w_{0}$ for positive (lop, $n>0$ ) and negative (bottom, $n<0$ ) dislocation dipoles. The numbers on the curves correspond to grain sizes of $D=1$ (solid circles), 10 (solid squares), and 100 (solid diamonds) $\mu \mathrm{m}$. (b) Wall exponent $n$ as a function of grain size for positive (top, $n>0$ ) and negative (bottom, $n<0$ ) dislocation dipoles with $d / w_{\sigma}=1$. The dash line indicates a low-temperature limit of wall exponents in large MD grains for temperatures below $200^{\circ} \mathrm{C}$.

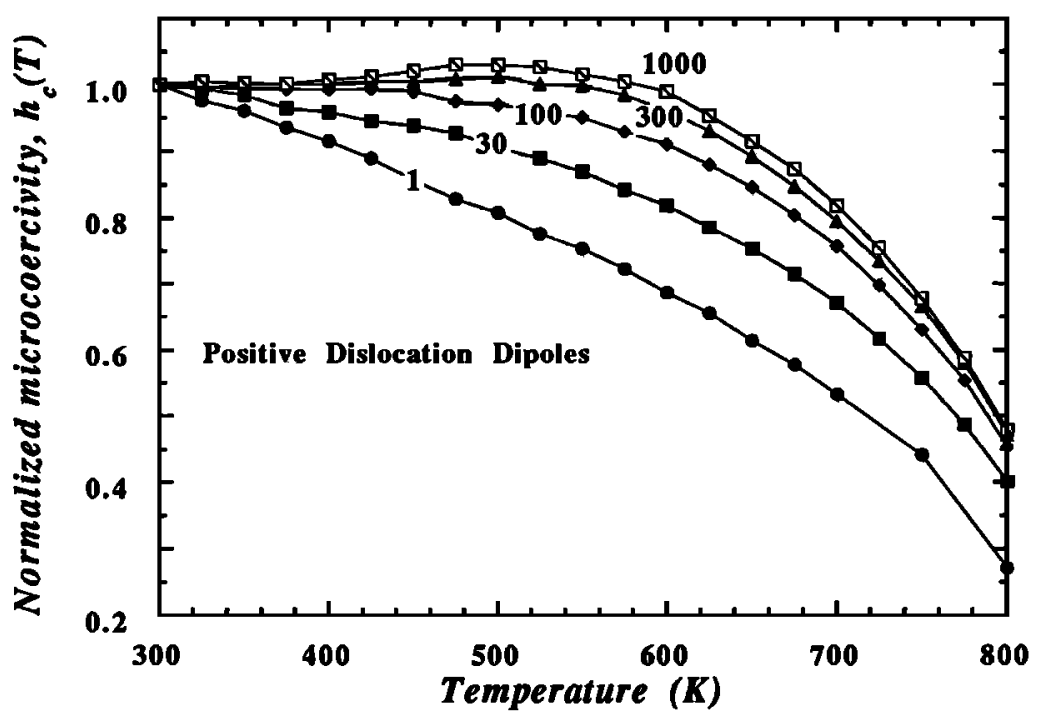

Fig. 5. Thermal dependence of normalized microcoercivity produced by pasitive dislocation dipoles with $d / w_{\sigma}=1$. The numbers on the curves are grain sizes in micrometers. 
ature dependence of $h_{c}(T)$ produced by planar defects with $d / w_{0}$ $=0.1$ and $D=10 \mu \mathrm{m}$ is shown in Figure 6 for a variety of different values of $A^{\prime} / A$ and $K_{u}{ }^{\prime} / K_{\mathrm{u}}$. The results can be grouped into three classes according to whether (1) exchange $\left(A^{\prime} / A \neq 1, K_{u}{ }^{\prime} / K_{u}=1\right)$, (2) anisotropy $\left(A^{\prime} / A=1, K_{u}{ }^{\prime} / K_{u^{\prime}} \neq 1\right)$, or (3) mixed $\left(A^{\prime} / A \neq 1, K_{u}^{\prime} / K_{u^{\prime}} \neq 1\right)$ pinning is operative. For exchange pinning, $h_{c}$ varies approximately as $h_{c}(T) \propto\left(A / M_{s}\right) w^{-3}$, whereas for anisotropy pinning, $h_{c}(T) \propto$ $\left(K_{u} / M_{s}\right) w^{-1.2}$. The mixed pinning case is a combination of the other two cases and $h_{c}(T)$ displays an intermediate dependence. Like the dislocation dipole models, the wall exponent for the exchange and anisotropy pinning models depends on $d / w_{0}$ as shown in Figure 7. The exponents for $d / w_{0}<1$ and $>1$ approach approximately the asymptotic solutions given by Hilzinger [1977]. Within each group shown in Figure 6, $h_{c}(T)$ dependence is identical but the magnitude of $h_{c}$ scales linearly with $A^{\prime} / A$ and $K_{u}{ }^{\prime} / K_{u}$ for $d / w_{0}<1$ (see Figure 8). Exchange pinning is more efficient than anisotropy pinning. At room temperature, a maximum microcoercivity of $\mu_{0} h_{\infty}=5.0 \mathrm{mT}$ occurs for $A^{\prime} / A=0$ and $K_{\mathrm{u}}{ }^{\prime} K_{\mathrm{u}}=0$ corresponding to a model for a nonmagnetic inclusion. This is about a factor of 2 larger than for a similar case of a positive dislocation dipole and almost 25 times larger than that for a negative dislocation dipole. The microcoercivity increases rapidly with $d / w_{0}$ [e.g., Aharoni, 1985]. For example, the same nonmagnetic inclusion with $d / w_{0}=10$ has $\mu_{0} h_{c}=33 \mathrm{mT}$. However, $h_{c}$ does not increase indefinitely but approaches a limiting value as $d / w_{0} \gg 1$ which depends on the ratios of $A^{\prime} / A$ and $K_{u}{ }^{\prime} / K_{u}$. An example is shown in Figure 9 for pinning models with a $\pm 5 \%$ change in $A$ and $K_{u}$. Within this regime, $h_{c o}$ has a similar magnitude to that calculated for negative dislocation dipoles but is a factor of 2-10 lower than for positive dipoles. This is an important distinction when considering combination models of dislocations and planar defects in the next section.

\section{Combination Models}

Combination models consist of a pair of dislocations bounding a planar defect. For instance, this configuration may be used to model the physical case in which perfect edge or screw dislocations dissociate into two partial dislocations with collinear Burgers vector separated by a stacking fault [e.g., Hull, 1975]. Experimental observations on $\mathrm{Ni}$-ferrite single crystals show that dissociation widths are 20-45 nm after high-temperature plastic deformation [Veyssiere et al., 1978]. If similar results hold for magnetite, calculations may be limited to models with $d w_{0}<1$. In the following, we will consider models with $d / w_{0}=0.1$ as being representative of this type of defect.

Model calculations can be divided into four cases depending on the sign of the dislocation dipole $(-$ or +$)$ and on the relative strength of the planar defect (strong or weak). The reason for this division according to the relative strength of the planar defect and the sign of the dipole is that the maximum pinning forces for a negative (positive) dipole and a strong (weak) planar defect add constructively but are opposed for a negative (positive) dipole and a weak (strong) planar defect. In some instances, this interplay results in complex behavior of $h_{c}(T)$.

The temperature dependence of $h_{c}(T)$ for a positive dipole bounding a planer defect is shown in Figure 10 for a range of relative strengths of the planar defect. For both exchange and anisotropy pinning, the thermal dependence of $h_{c}(T)$ is not much different from that of the dislocation dipole model alone until the strength of the defect exceeds $\pm 20 \%$. Room temperature microcoercivity for the positive dislocation dipole, which acts like a simple double dislocation for $d / w_{\sigma}=0.1$, is 2-10 times larger than the corresponding $\boldsymbol{h}_{\mathrm{c}}$ for either a strong or weak planar defect. Therefore, the magnetoclastic effects of the dipole dislocations overwhelm the effects of the planar defect over the parameter space shown in Figure 10. This could partly explain wall pinning at stacking faults in magnetite observed by Jakubovics et al. [1978] if the stacking faults are bounded by dislocations.

The temperature dependence of $h_{c}(T)$ for a negative dipole bounding a strong planar defect is shown in Figure 11. Here, the effects of the planar defect become important after only a few percent change in the strength of the defect. A negative dislocation dipole with $d / w_{o}=0.1$ has an $h_{c o}$ that is of the same magnitude as $h_{\infty}$ produced by the planar defect. The pinning forces resulting from a negative dipole combined with a strong planar defect have the same sign and add constructively so that the $h_{c}(T)$ curves shown in Figure 11 transform gradually from dislocation

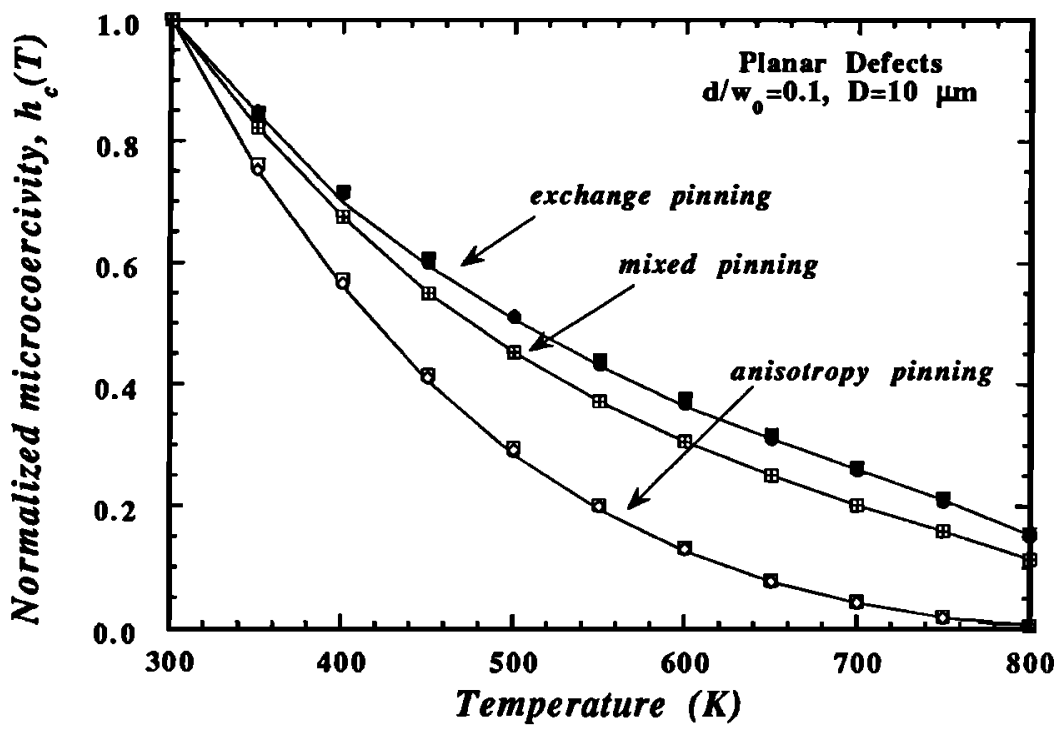

Fig. 6. Thermal dependence of normalized microcoercivity produced by planar defects with $d / w_{o}=0.1$ and $D=10 \mu m$. Exchange pinning models (soild symbols) are $A^{\prime} / A=0.9,0.5$, and 0 ; anisotropy pinning models (open symbols) are $K_{u}{ }^{\prime} / K_{u}=0.9,0.5$, and 0 ; and, mixed pinning model (hatched symbol) is $K_{u}{ }^{\prime} / K_{u}=0$ and $A^{\prime} / A=0$. The solid lines for exchange and anisotropy pinning models correspond to the thermal dependence of $\left(A / M_{s}\right) w^{-3}$ and $\left(K_{x} / M_{3}\right) w^{-12}$, respectively. 


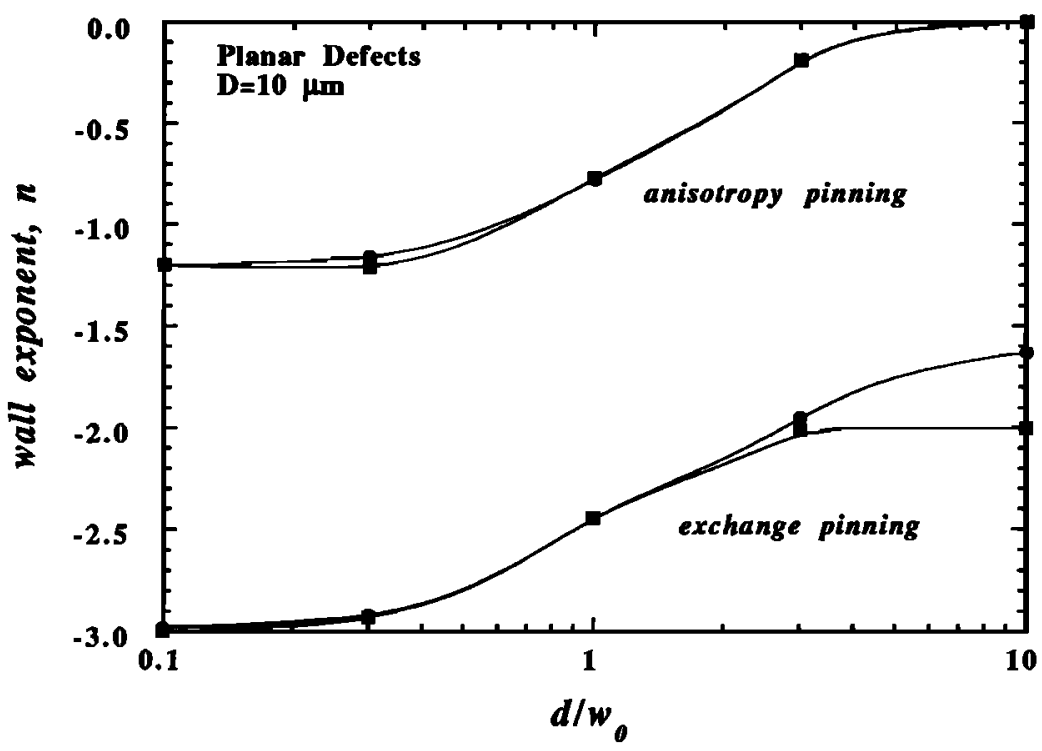

Fig. 7. Wall exponent as a function of $d / w_{0}$ for planar defects with exchange and anisotropy pinning. Solid circles correspond to weak planar defects, and solid squares correspond to strong planar defects.

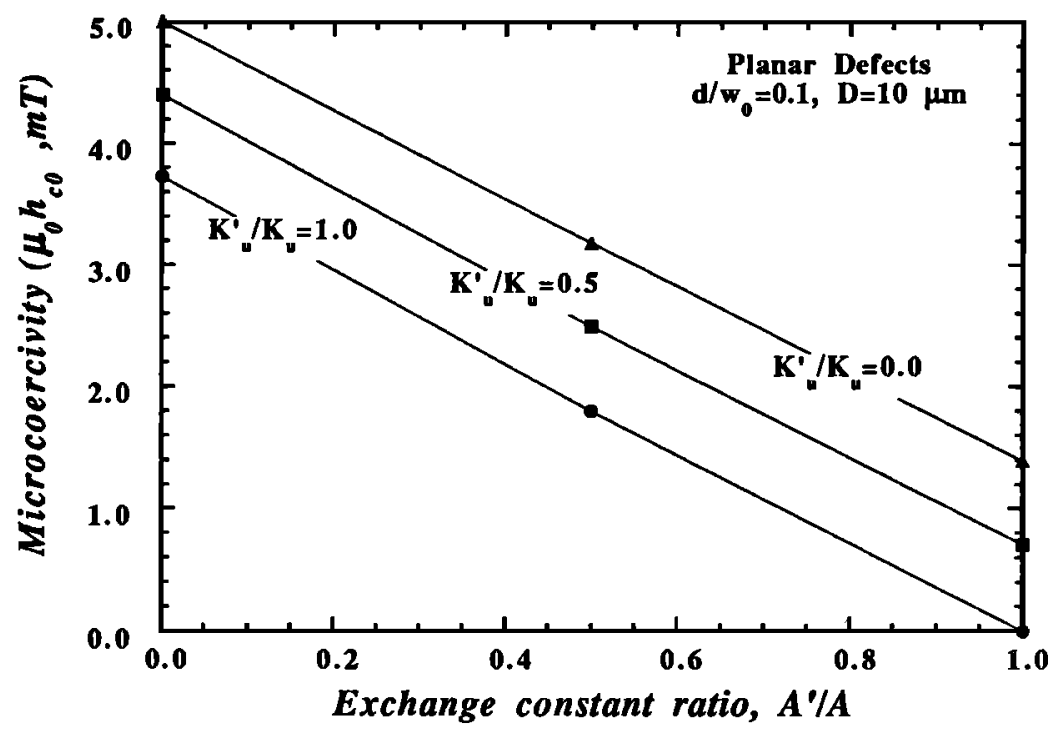

Fig. 8. Microcoercivity as a function of $A^{\prime} / A$ for constant values of $K_{u}{ }^{\prime} / K_{u}=1.0,0.5$ and 0.0 . Model parameters are $d / w_{\sigma}=0.1, D=10$ $\mu \mathrm{m}$, and $\mathrm{T}=300 \mathrm{~K}$.

dipole pinning to exchange or anisotropy pinning as the relative strength of the planar defect increases.

The final case modeled consists of a negative dipole combined with a weak planar defect. In this regime, the pinning forces of the dislocations and planar defect have opposite signs and add destructively for certain parameter and temperature ranges. Some extreme examples are shown in Figure 12 for anisotropy pinning. Because of the difference between the temperature dependence of $K_{z}$ and $\lambda_{11}$, the anisotropy pinning force drops much faster with temperature than the dislocation pinning force. Hence, over a particular temperature range, because the two pinning forces bave opposite signs, the total pinning force begins to increase, resulting in an increase in the microcoercivity. Exchange pinning models produce results that are not as complex as the anisotropy pinning models because of the similar thermal dependence of exchange and magnetostriction constants. Overall, $h_{d}(T)$ does not fall between the limits for dislocation dipole only and planar defect only models as it does for positive dipoles (see Figure 11 ).

\section{Discussion}

The micromagnetic calculations predict the temperature dependence of microcoercivity as a function of grain size, domain wall width, defect spacing, and type of defect. For most types of defects considered, $h_{c}(T)$ is a function of the wall width. However, the exact wall width dependence is determined by the defect spacing, particularly for models involving dislocations. The microcoercivity produced by a dislocation dipole can be described simply by the quantity $\left(\lambda / M_{s}\right) w(T)^{n}$, where $n$ varies from +0.6 to -1 depending on the value of $d / w_{0}$. Expressions for $h_{c}(T)$ appropriate for dislocation dipoles and planar defects are listed in Table 2.

The model calculations are valid for the interaction of a single defect with a single domain wall. In most instances, this configuration is probably an unrealistic model for magnetite grains in a rock. To relate $h_{c}(T)$ to the bulk or macroscopic coercivity, $H_{c}$, one needs to account for the interactions of many defects with many walls [e.g., Träuble, 1969; Xu and Merrill, 1990a]. This is 


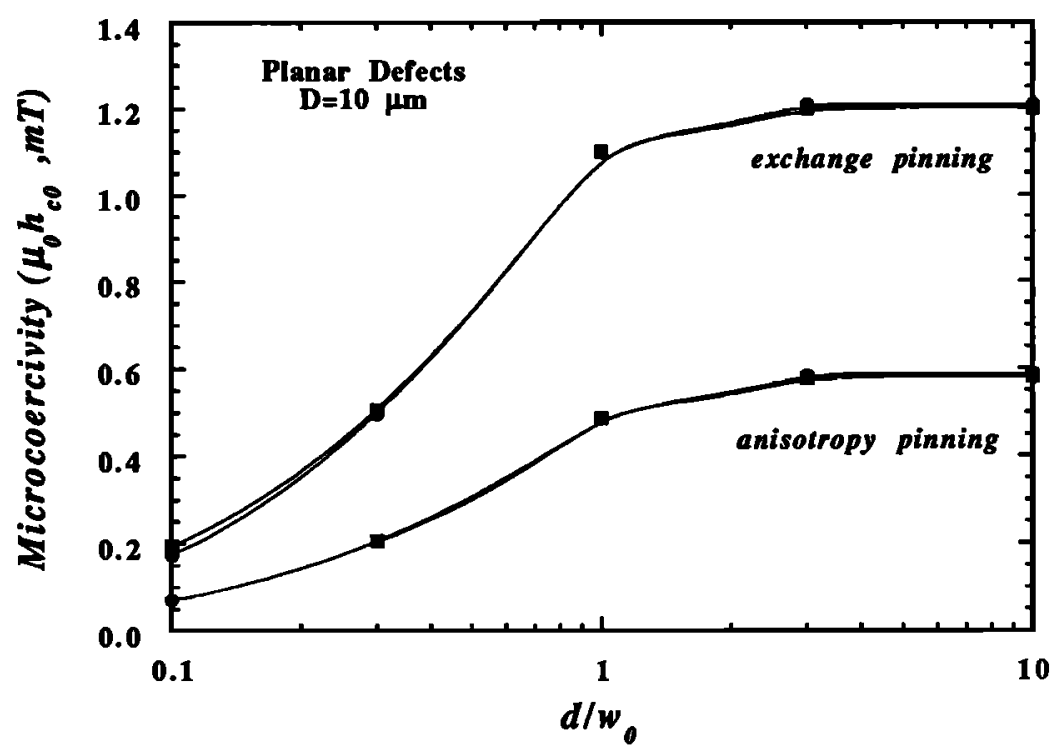

Fig. 9. Microcoercivity as a function of $d / w_{0}$ for planar defects with exchange and anisotropy pinning. Solid circles correspond to weak planar defects $\left(A^{*} / A\right.$ or $\left.K_{4}{ }^{\prime} / K_{4}=0.95\right)$, and solid squares correspond to strong planar defects $\left(A^{\prime} / A\right.$ or $\left.K_{4}{ }^{\prime} / K_{4}=1.05\right)$. Model parameters are $D=10 \mu \mathrm{m}$ and $\mathrm{T}=300 \mathrm{~K}$.
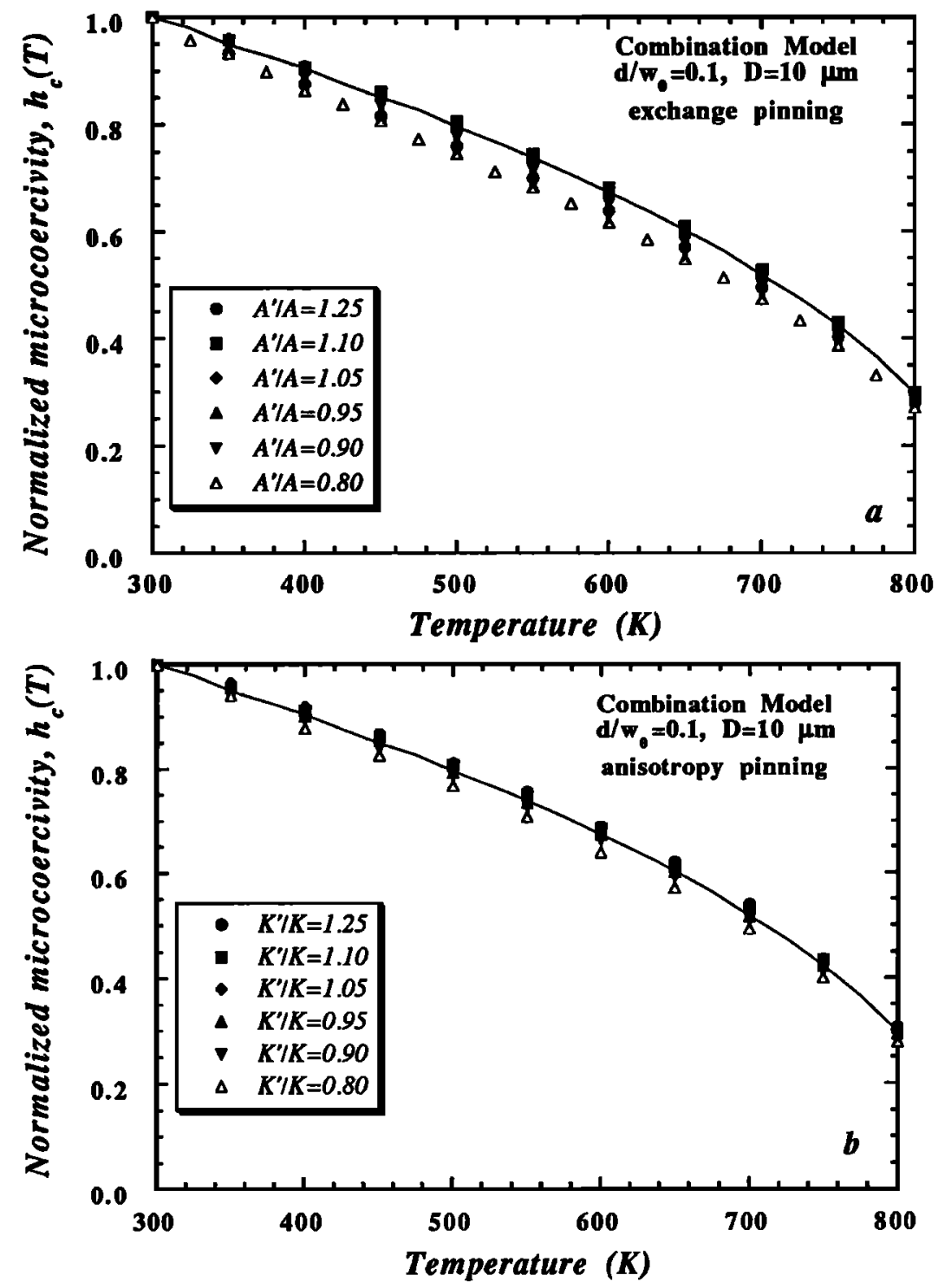

Fig. 10. Thermal dependence of normalized microcoercivity produced by a planar defect with (a) exchange pinning or $(b)$ anisotropy pinning bounded by a positive dislocation dipole. Model parameters are $d / w_{0}=0.1$ and $D=10 \mu \mathrm{m}$. Exchange and anisotropy pinning values are given in the figure. The solid line corresponds to the thermal dependence of $\lambda / M_{\text {s. }}$ Note that $K^{\prime} / K=K_{\triangleleft}{ }^{\prime} / K_{\text {s. }}$. 

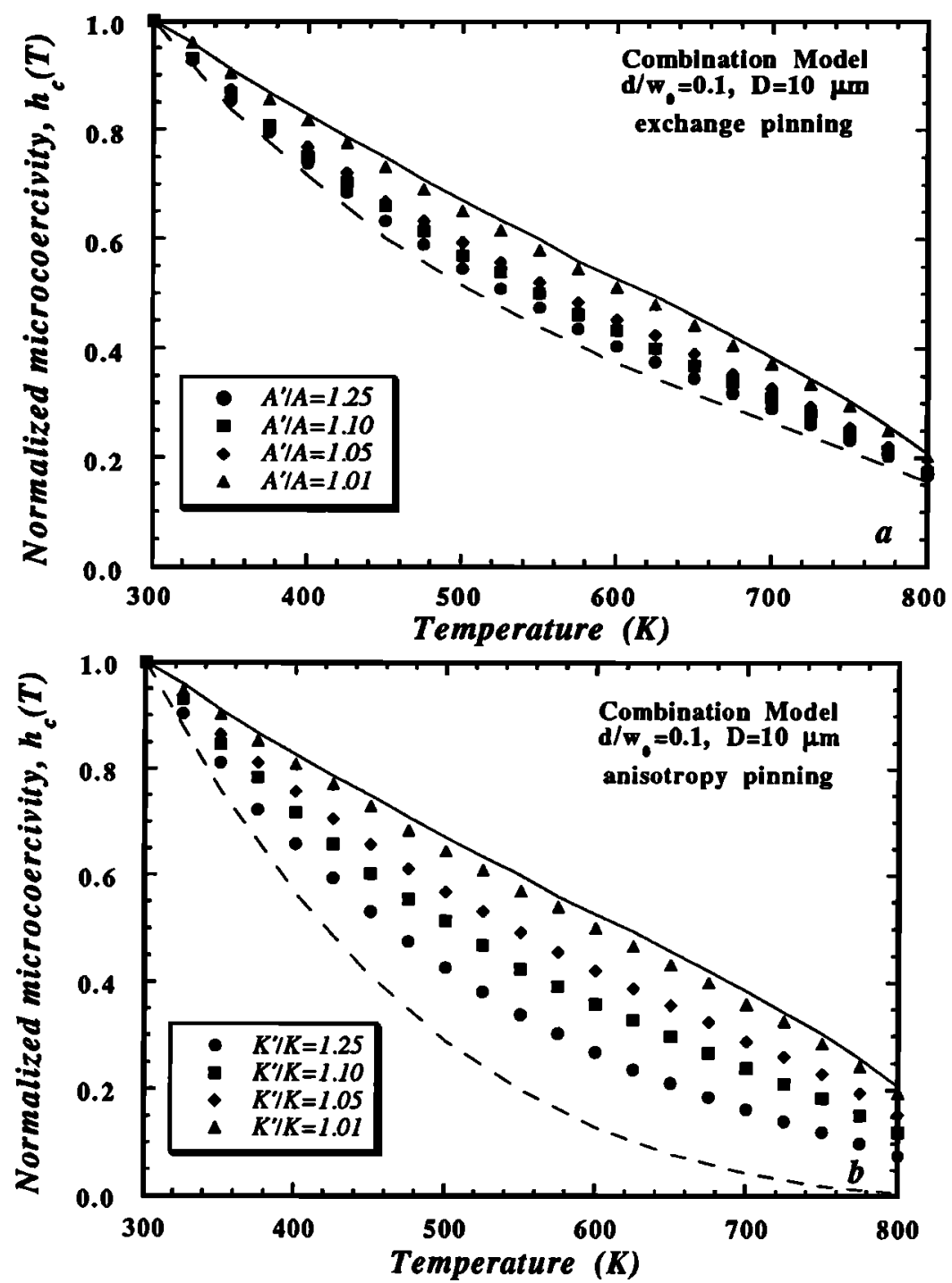

Fig. 11. Thermal dependence of normalized microcoercivity produced by a strong planar defect with $(a)$ exchange pinning or $(b)$ anisotropy pinning bounded by a negative dislocation dipole. Model parameters are $d / w_{\sigma}=0.1$ and $D=10 \mu \mathrm{m}$. Exchange and anisotropy pinning values are given in the figure. The solid line corresponds to the thermal dependence of $\left(\lambda / M_{s}\right) w^{-1}$, and the dashed line corresponds to the thermal dependence of $(a) A M M_{s} w^{-3}$ and $(b) K_{*} / M_{s} w^{-1.2}$. Note that $K^{\prime} / K=K_{s}^{\prime} / K_{*}$.

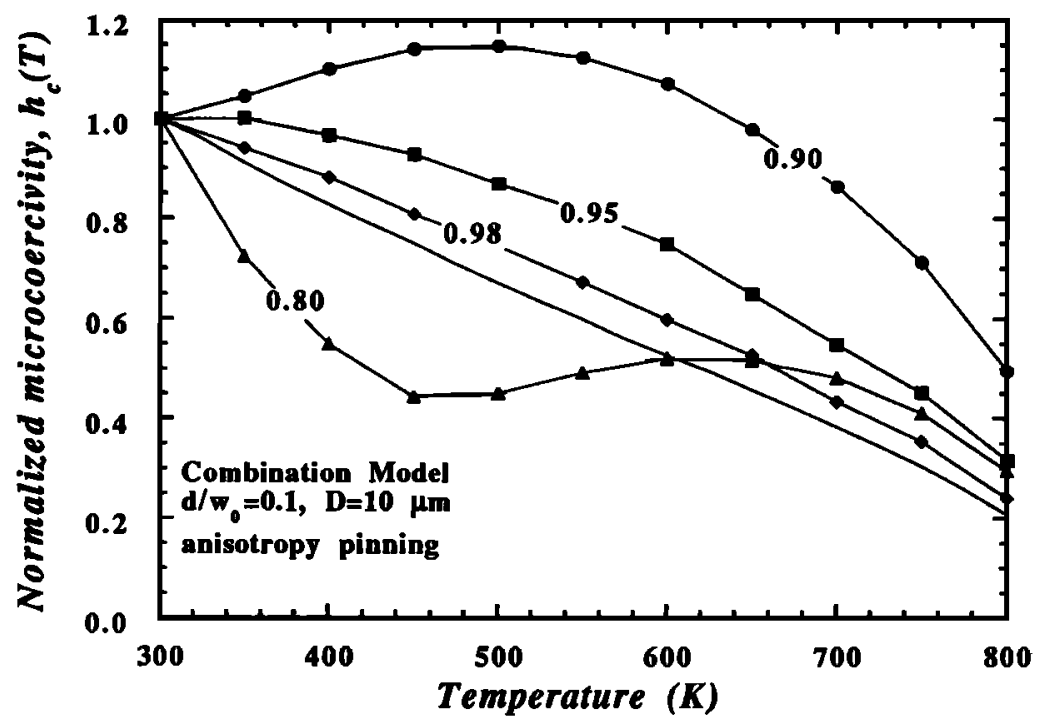

Fig. 12. Thermal dependence of normalized microcoercivity produced by a weak planar defect with anisotropy pinning bounded by a negative dislocation dipole. Model parameters are $d / w_{0}=0.1$ and $D=10 \mu \mathrm{m}$. The numbers on the curves indicate $K_{\star}{ }^{\prime} / K_{u}=0.80$, $0.90,0.95$, and 0.98 . The solid line corresponds to the thermal dependence of $\left(\lambda / M_{s}\right) w^{-1}$. 
TABLE 2. Thermal Dependence of Microcoercivity for Various Types of Defects

\begin{tabular}{|c|c|c|c|}
\hline $\begin{array}{c}\text { Type of } \\
\text { Defect }\end{array}$ & $\begin{array}{c}\text { Thermal Dependence } \\
\text { of } h_{c}^{*}\end{array}$ & $\begin{array}{c}\text { Wall Exponent, } \\
n\end{array}$ & $\begin{array}{c}\text { Parameter } \\
\text { Range }\end{array}$ \\
\hline \multicolumn{4}{|c|}{ Dislocations } \\
\hline Single dislocation & $\lambda / M$ & $n=0$ & $d / w_{\infty}>1$ \\
\hline Negative dislocation dipole & $\left(\lambda / M_{s}\right) w^{n}$ & $-1 \leq n \leq 0$ & $0.1 \ll<d / w_{0}<+\infty$ \\
\hline Positive dislocation dipole & $\left(\lambda / M_{s}\right) w^{n}$ & $\begin{array}{c}0 \leq n \leq 0.6 \\
n=0\end{array}$ & $\begin{array}{c}0.1<<d / w_{0} \leq 1 \\
d / w_{0}>>1\end{array}$ \\
\hline \multicolumn{4}{|c|}{ Planar Defect } \\
\hline Exchange pinning & $\left(A / M_{s}\right) w^{n}$ & $-3<n<-2$ & $0.1<<d / w_{0}<+\infty$ \\
\hline Anisotropy pinning & $\left(K_{u} / M_{s}\right) w^{n}$ & $-1.2<n<0$ & $0.1<<d / w_{0}<+\infty$ \\
\hline \multicolumn{4}{|c|}{ Stacking Fault } \\
\hline $\begin{array}{l}\text { Positive dipoles } \\
\text { strong, weak defect }\end{array}$ & $\approx \lambda / M_{s}$ & $n=0$ & $\begin{array}{c}d / w_{o}=0.1 \\
A^{\prime} / A, K_{\mathrm{u}} / K_{\mathrm{u}}<20 \%\end{array}$ \\
\hline $\begin{array}{l}\text { Negative dipoles } \\
\text { strong defect }\end{array}$ & $\approx\left(\lambda / M_{s}\right) w^{n}$ & $n=-1$ & $\begin{array}{c}d / w_{0}=0.1 \\
A^{\prime} / A, K_{4} / K_{4}<5 \%\end{array}$ \\
\hline
\end{tabular}

an especially difficult problem to solve in even an approximate way. In a first order theory, $X u$ and Merrill [1990a] have suggested that the relationship between $H_{c}$ and $h_{c}$ follows one of two limiting cases depending on defect density

$$
\begin{aligned}
\text { Low defect density } & H_{c} \propto \frac{h_{c}^{2}}{M_{s}} \\
\text { High defect density } & H_{c} \propto<h_{c}>
\end{aligned}
$$

where $\left\langle h_{c}\right\rangle$ is the mean microcoercivity and must be derived separately. In one such derivation [Träuble, 1969; Xu and Merrill, $1990 a, 1992]$, the root mean square microcoercivity for a large number $m$ of randomly distributed identical defects is

$$
<h_{c}>\propto \sqrt{m w} h_{c}
$$

Using (23), $H_{c}(T)$ can be calculated from the model $h_{c}(T)$ results produced by different types of defects. However, it should be remembered that (23) is only a first-order approximation for macroscopic coercivity and its application to synthetic or natural samples will be mitigated by such things as grain size distribution effects, transdomain processes, thernal fluctuations. and thermal diffusion of defects. Another basic limitation of this approach is that the theoretical models are one dimensional whereas macroscopic coercivity may involve mechanisms that are inherently three dimensional. With these caveats in mind, in the next two sections, we compare the micromagnetic calculations to published experimental $H_{c}(T)$ data for small MD magnetite grains $(\langle D\rangle \approx 1$ $20 \mu \mathrm{m})$ with low and high defect densities.

\section{Low Defect Density}

The low defect density (LDD) approximation was derived by assuming an assemblage of two-domain grains with one identical defect per grain [Xu and Merrill, 1990a]. $H_{c}(T)$ calculated using the LDD approximation and the model results for a single dislocation (curve 2), negative dipole (curves 3 and 4), positive dipole (curves 1 and 2), planar defect (curves 5 and 6), and two parallel dislocations bounding a stacking fault (curve 2) is shown in Figure 13 along with experimental coercivity data for magnetite. The experimental data were taken from published results from recrystallized synthetic magnetite produced either by high-temperature hydrothermal recrystallization [Heider et al., 1987] or by lowtemperature decomposition of $\mathrm{FcO}$ [Dunlop and Bina. 1977]. The recrystallized synthetic magnetites are helieved to have low residual strains and a low defect density [Heider et al., 1987]. If the LDD approximation is valid for these samples, then a negative dislocation dipole model with $0.1<d / w_{0}<1$ provides a reasonable fit to the experimental data. Planar defects, positive dipoles, and single dislocations produce an $H_{c}(T)$ that varies either too rapidly or too slowly with temperature compared with the experimental data. The individual dislocations of a positive dipole, being mutually repulsive, would tend to find equilibrium positions as far apart from one another as possible $\left(d / w_{0}>1\right)$, i.e., would no longer behave as a dipole. Hence. for low defect density, a positive dipole acts like a single dislocation in controlling the microcoercivity. The differences between the two experimental data sets can be partly attributed to the different stress-strain histories of the samples and associated differences in defect type and geometry. Models that describe grains having a single dislocation produce a poor fit to the experimental data (Figure 13, curve 2), which is contrary to the conclusion reached by $X u$ and Merrill [1990a]. The discrepancy results from the temperature dependence assumed for the magnetostriction constant. $X u$ and Merrill [1990a] obtain a reasonably good fit to the experimental data with a single dislocation model by using the experimentally determined magnetostriction values from Klapel and Shive [1974]. Recent and more extensive measurements of magnetostriction by Moskowitz [1993] show that $\lambda$ does not decrease with temperature as rapidly as the earlier results obtained by Klapel and Shive [1974]. For example, $\lambda \propto M_{s}^{2.3}$ for the new measurements compared with $M_{s}^{2.6}$ for the older measurements. Although this difference appears minor, it becomes significant when substituted into the LDD approximation, $H_{c}(T) \propto$ $\lambda^{2} / M_{s}^{3}$, for single dislocations. Therefore, instead of $H_{c}(T) \propto M_{s}^{2.2}$, the new experimental determination of $\lambda(T)$ predicts that $H_{c}(T)$ varies approximately as $M_{s}^{1.6}$.

Using the new magnetostriction data, the present results suggest that wall pinning in small MD recrystallized magnetite grains is due to a few negative dislocation dipoles instead of noninteracting single dislocations. This is a subtle distinction, but some indirect evidence to support this interpretation is provided by Smith and Merrill [1983]. On the basis of domain wall resonance experiments, they suggested that the likeliest pinning sites in some natural magnetites may be dislocation dipoles, or some other dislocation array, rather than isolated single dislocations. 


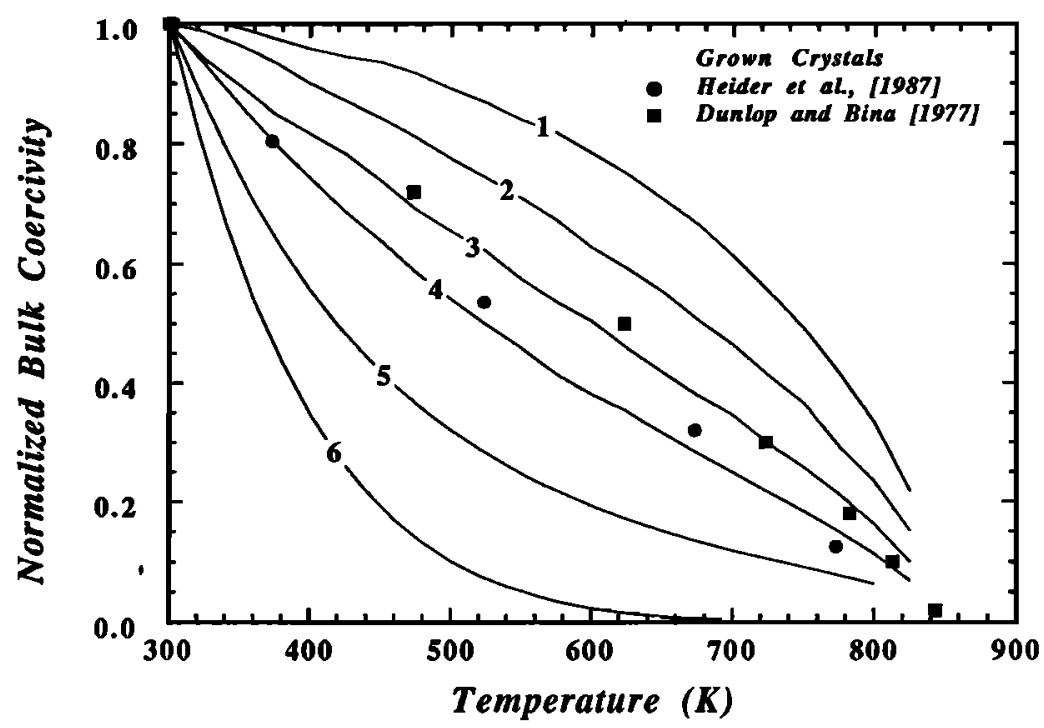

Fig. 13. Comparison of experimental and theoretical thermal dependence of normalized bulk coercivity, $H_{c}(T) / \mathrm{H}_{c 0}$ produced by different types of defects, using low defect density approximation. Numbers on the curves correspond to the following defect geometries: (1) positive dislocation dipole, $d / w_{o}=1, D=10 \mu \mathrm{m}$, (2) single dislocalion, positive dislocation dipole, $d / w_{0}=0.1$, or positive dislocation dipole bounding a stacking fault, $d / w_{0}=0.1$, (3) negative dislocation dipole, $d / w_{0}=1$, (4) negative dislocation dipole, $d / w_{\sigma}=0.1,(5)$ planar defect with exchange pinning, $d / w_{0}=0.1$, and $(6)$ planar defect with anisotropy pinning, $d / w_{0}=0.1$. Grain size is $D=10 \mu \mathrm{m}$. Experimental data are for grown crystals of synthetic magnetite with average grain sizes of $D \approx 12 \mu \mathrm{m}$ [Heider et al., 1987] and $D \approx 1-5 \mu \mathrm{m}$ [Dunlop and Bina, 1977].

\section{High Defect Density}

The high defect density (HDD) approximation may apply to magnetite grains that have been crushed from larger crystals [Dankers and Sugiura, 1981], glass-ceramic samples that have been quenched from high temperature [Worm et al., 1988], and natural samples, all which are likely to have substantial concentrations of defects. For example, crushed grains that have been milled have probably undergone extensive brittle/plastic deformation at low temperature, resulting in a rapid increase in the number of defects and a high defect density. Under these conditions, the experimental thermal dependence of the macroscopic coercivity varies approximately as $\lambda / M_{s}$ and apparently is independent of any changes in wall width with temperature [Hodych, 1982; Xu and Merrill, 1992].

To predict the macroscopic coercivity of such samples under the HDD approximation, the mean microcoercivity must be calculated (see equation (23)). In one case, $\left\langle h_{c}\right\rangle$ for a large number of randomly distributed identical defects, for example, dislocation dipoles all with the same value of $d / w_{0}$, can be calculated from (24) and the results in Table 2 . Still, only negative dipoles with $d / w_{0} \approx 1$ will produce $\left\langle h_{c}\right\rangle$ independent of wall width and give the experimental observation that $H_{c}(T) \circ \lambda / M_{s}$ [e.g., $X u$ and Merrill, 1992]. In another approach, to obtain the simple $\lambda / M_{s}$ behavior, $X u$ and Merrill [1992] suggest that $\left\langle h_{c}\right\rangle$ can be attributed to a general sinusoidal microstress field which is produced by dislocations and is further assumed to affect the wall anisotropy in such a way as to produce a wall width nearly independent of temperature.

While the arguments in the preceding paragraph may apply under special circumstances to specific defect type or geometry, it is not a satisfactory explanation for coercivity in MD grains that may contain a variety of defect types and arrangements. For the important case of dislocations, a MD grain may more plausibly be expected to contain both positive and negative dislocation dipoles with a range of $d / w$. A random distribution of dislocations produces a corresponding distribution of interaction widths that produces finally a distribution of wall width exponents. Model calculations predict that $h_{\epsilon}$ resulting from dislocation dipoles will exhibit both positive and negative values for the wall exponent. For positive wall exponents, the thermal dependence of $h_{c}$ varies with temperature more slowly than $\lambda / M_{s}$, whereas, for negative wall exponents, $h_{c}$ varies faster than $\lambda / M_{s}$. Hence, the mean microcoercivity from such a distribution should depend less on the thermal expansion of the wall than for models that include a random distribution of only one type of defect (ie., $d / w_{0}=$ constant). As a simple example, the mean micrococrcivity is equated to the average value of $h_{c}$ determined by summing the contributions from equally weighted pairs of positive and negative dislocation dipoles with discrete values of $d / w_{0}(0.1,0.5,1,2,5$, 10). The result of this calculation is given in Figure 14 for $D=10$ $\mu \mathrm{m}$ and shows that for this case, $\left\langle h_{c}\right\rangle \approx \lambda / M_{s}$. A weighted average based on the magnitudes of $h_{c}$ 's gives essentially the same result. In the limit for a large number of positive and negative dipoles with a random distribution of $d / w_{0}$, this result should still be a good approximation for two reasons: Firstly, when $d / w_{0}>1$, the wall exponents for positive and negative dipoles rapidly approach zero (see Figure $4 a$ ) and $h_{c}$ will be independent of the thermal expansion of the wall width; secondly, when $d / w_{0}<1, n \rightarrow 0$ for positive dipoles and -1 for negative dipoles. However, within this range of $d w_{0}$, the magnitude of $h_{c}$ for positive dipoles is at least twice that for negative dipoles. Therefore, the microcoercivity for $d / w_{0}<1$ will be dominated by positive dipoles and $h_{c}$ will again be independent of the thermal expansion of the wall width. For large MD grains with $D>100 \mu \mathrm{m}$, the simple averaging scheme leading to $\left\langle h_{c}\right\rangle \approx \lambda / M_{s}$ may no longer hold because the wall width expands rapidly with temperature, producing more complex $h_{c}(T)$ behavior for positive dipoles (see Figure 5).

\section{Conclusions}

A one dimensional micromagnetic model was formulated to calculate the thermal dependence of microcoercivity for several different types of defects. Within the approximations of the model, 


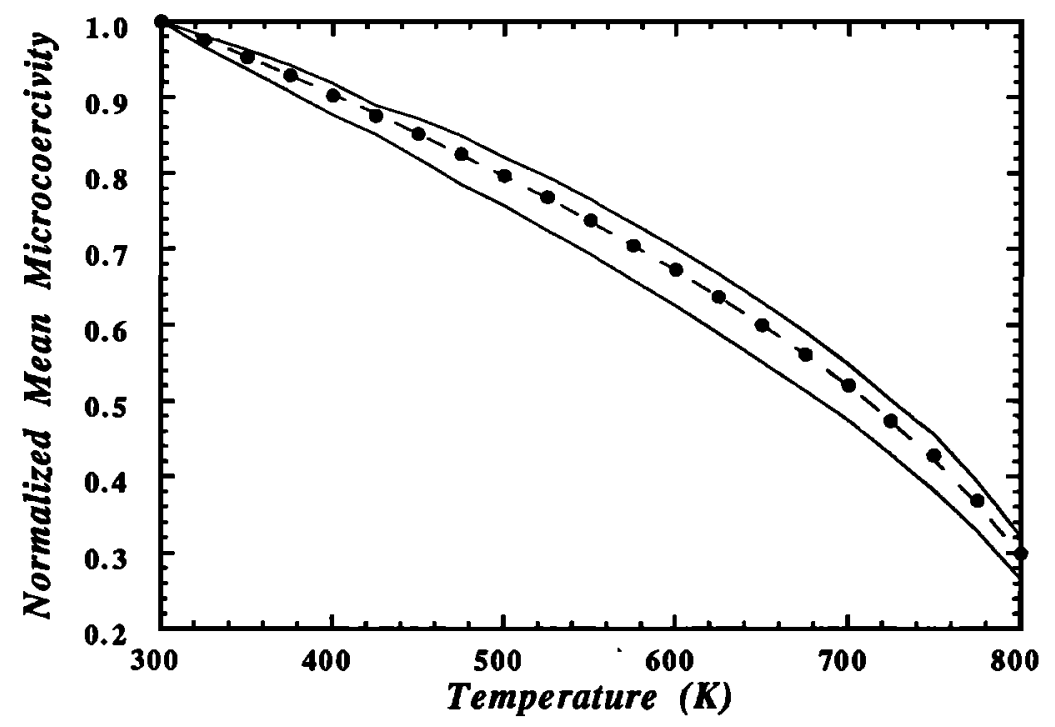

Fig. 14. Normaljzed thermal dependence of mean microcoercivity produced by an assemblage of negative and positive dislocation dipoles with $d / w_{0}=0.1,0.5,1,2,5$, and 10. Grain size is $D=10 \mu \mathrm{m}$. Upper solid curve is $\left\langle h_{c}\right\rangle$ for positive djpoles, lower solid curve is $\langle h\rangle$ for negative dipoles, solıd circles are $\langle h\rangle$ for both negative and positive dipoles, and the dashed line is the thermal dependence of $\lambda / M_{s}$.

$h_{c}(T)$ is usually found to be a function of the wall width raised to some power $n$. The particular value of $n$ is found to be a function of the DW-defect interaction spacing $\left(d / w_{0}\right)$, type of defect, and grain size. The thermal dependence of the wall width is a natural outcome of the calculations. Within the grain size range of 1 to $100 \mu \mathrm{m}$, the wall width expands with temperature more gradually than classical theory predicts. Only for large MD grains $(D>100$ $\mu \mathrm{m}$ ) does the wall width expansion approach the classical limit. The reduced expansion of the wall width is due to the magnetostatic energy of the wall and not to any direct influence of the defect on the wall anisotropy.

The model results for microcoercivity were used with the theory of $X u$ and Merrill [1990a] to relate microcoercivity to the observed macroscopic coercivity. Results for dislocation dipoles provide the best agreement with the limited experimental data available for magnetite. Dipoles are considered here in a general sense as two associated dislocations interacting with a domain wall. For grains with low defect densities, i.e., hydrothermally grown magnetite, negative dislocation dipoles with $d / w_{0} \approx 0.1-1$ have a theoretically calculated thermal dependence of coercivity that agrees well with experimental results [Heider et al., 1987]. In the high defect density limit, a distribution of positive and negative dislocation dipoles with a distribution of dipole widths yields $H_{c}(T) \propto \lambda / M$, consistent with experimental data from crushed and glass ceramic samples. However, without specific observations of the defect structures that are typical in recrystallized and crushed magnetites, it is difficult to use the theoretical results to make unambiguous statements about the importance of various defect structures in real samples. Coercivity measurements from single grains represent one instance in which the theoretical microcoercivity results could be applied directly [e.g., Halgedahl, 1991; Heider and Hoffmann, 1992], since grain size distribution effects are eliminated.

The other main conclusions are as follows:

1. The thermal dependence of microcoercivity produced by dislocations can be given as $h_{c}(T) \propto\left(\lambda / M_{s}\right) w(T)^{\prime \prime}$. The wall width exponent $(n)$ is a function of $d / w_{o}$ and grain size.

2. For negative dislocation dipoles, $h_{c}(T)$ varies inversely with the wall width. The wall width exponent varies from -1 for $d / w_{0}$ $\ll 1$ to 0 for $d / w_{0}>1$.

3. For positive dislocation dipoles, $h_{c}(T)$ varies directly with $w$.
The wall width exponent reaches a maximum value of approximately 0.6 for $d / w_{0} \approx 1$ and approaches zero for $d / w_{0}<<1$ and $d / w_{0}$ $\gg 1$. When the grain size is larger than $100 \mu \mathrm{m}$ and $d / w_{0} \approx 1$, the expansion of the wall width outpaces the decrease in $\lambda / M$, over a limited temperature range, resulting in a nearly constant $h_{c}(T)$ for $\mathrm{T}<200^{\circ} \mathrm{C}$ and reaching a maximum between $200-300^{\circ} \mathrm{C}$.

4. The thermal dependence of $h_{c}$ for planar defects can be divided into three regimes independent of the relative change in the exchange or anisotropy constants within the defect region: (1) exchange pinning regime in which $h_{c}(T) \propto\left(A / M_{\mathrm{s}}\right) w(T)^{n}$ with $-3<n<-2$; (2) anisotropy pinning regime in which $h_{c}(T) \propto\left(K_{u} / M_{s}\right) w(T)^{n}$ with $-1.2<n<0$; and (3) mixed pinning regime which is a combination of types 1 and 2 and cannot be described by a simple power law dependence on the wall width. The absolute magnitude of $h_{c}$, however, is a very strong function of the strength of the defect. All planar models produce a more rapid decrease in $h_{c}$ with temperature than dislocation models.

5. The thermal dependence of $h_{c}$ produced by a planar defect bounded by a positive dislocation dipole and $d / w_{0}=0.1$ is approximately the same as the dislocation dipole alone until the relative strength of the planar defect exceeds $20 \%$. For this defect geometry, the magnetoelastic effects of the dislocations are much greater than the pinning effects of the planar defect.

6. The thermal dependence of $h_{c}$ produced by a planar defect bounded by a negative dislocation dipole and $d / w_{0}=0.1$ is more complex than the defect geometry discussed in conclusion 5 because of the competing effects of the dislocations and planar defect. The effect of the planar defect becomes important after only a $5 \%$ change in its relative strength. For strong planar defects, $h_{c}(T)$ behavior transforms gradually from dislocation dipole pinning to planar defect pinning as the relative strength of the defect increases. For weak planar defects, complex $h_{c}(T)$ behavior is predicted.

\section{APPENDIX}

The complete expressions for the two nonlinear equations given by (19) are

$$
\frac{\partial E_{t}}{\partial x_{0}}=0
$$




$$
\begin{gathered}
\frac{(1+\tau)}{8}(1+\Lambda)\left[C^{\prime}\left(\frac{d-x_{0}}{w}\right)-C^{\prime}\left(\frac{x_{0}}{w}\right)\right] \\
+\left(\frac{d}{4 T w}\right)^{2}(1-\Omega)\left[E^{\prime}\left(\frac{d-x_{0}}{w}\right)-E^{\prime}\left(\frac{x_{0}}{w}\right)\right] \\
+\frac{\sigma}{2}\left\{\left[2 \sqrt{2} C^{\prime}\left(\frac{x_{0}}{w}\right)-B^{\prime}\left(\frac{x_{0}}{w}\right)\right] \pm\left[2 \sqrt{2} C^{\prime}\left(\frac{d-x_{0}}{w}\right)+B^{\prime}\left(\frac{d-x_{0}}{w}\right)\right]\right\}=h \\
\frac{\partial E_{t}}{\partial w}=0 \\
\frac{\pi}{2 Q}\left[\frac{2 w}{D} \ln \left(1+\frac{D}{w}\right)-\frac{w}{w+D}\right]+\frac{\pi}{2}(1+\tau)-2 \pi(\sqrt{2}-1)\left(\frac{d}{T w}\right)^{2} \\
\quad-\frac{(1+\tau)(1-\Lambda)}{2}\left[G\left(\frac{d-x_{0}}{w}\right)+G\left(\frac{x_{0}}{w}\right)\right] \\
+\frac{\sigma}{2}\left\{\left[2 \sqrt{2} G\left(\frac{d}{w}\right)^{2}(1-\Omega)\left[F\left(\frac{d-x_{0}}{w}\right)+F\left(\frac{x_{0}}{w}\right)\right]\right.\right. \\
\left.\left.+L\left(\frac{x_{0}}{w}\right)\right] \pm\left[2 \sqrt{2} G\left(\frac{d-x_{0}}{w}\right)+L\left(\frac{d-x_{0}}{w}\right)\right]\right\}=0
\end{gathered}
$$

where

$$
\begin{aligned}
B^{\prime}(x) & =\frac{-2 x \sqrt{2+x^{2}}}{\left(1+x^{2}\right)^{2}}, \quad C^{\prime}(x)=\frac{2}{\left(1+x^{2}\right)^{2}}, \quad E^{\prime}(x)=\frac{2}{\left(1+x^{2}\right)^{2}\left(2+x^{2}\right)} \\
F(x) & =E(x)+x E^{\prime}(x), G(x)=C(x)-x C^{\prime}(x), L(x)=B(x)-x B^{\prime}(x)
\end{aligned}
$$

and $\Lambda=K_{u}{ }^{\prime} / K_{u}, \Omega=A^{\prime} / A, \tau=27 \mu \lambda_{11} / 4 K_{l}, T=d\left(K_{u} / A\right)^{\prime h}, Q=K_{u} / 2 \pi M_{s}{ }^{2}$, $h=H M_{s} / 2 K_{v}$, and $\sigma=\lambda_{H} b \mu / D K_{u}$,

Acknowledgments. I thank R. Merrill, S. Xu, and B.J. Wanamaker for helpful comments and discussions. This research was supported by National Science Foundation grant EAR-9017389. Support for the IRM is provided by grants from the Keck Foundation and the National Science Foundation. This is contribution $\mathbf{9 2 0 6}$ of the Institute for Rock Magnetism.

\section{REFERENCES}

Aharoni, A., Domain wall pinning al planar defects, J. Appl. Phys., 58 , 2677-2680, 1985.

Borradaile, G.J., Experimental deformation of two-component IRM in magnetite-bearing limestone: a model for the behavior of NRM during natural deformation, Phys. Earth Planet. Inter., 70, 64-77, 1992.

Cullity, B.D., Introduction to Magnetic Materials, 666 pp., AddisonWesley, Reading, Mass., 1972.

Dankers, P., and N. Sugiura, The effects of annealing and concentration on the hysteresis properties of magnetite around the PSD-MD transition, Earth Planet. Sci. Lett., 56, 422-428, 1981.

Dietze, H.D., and H. Thomas, Bloch- und Néel-Wände in dünnen ferto magnetischen Schichten, Z. Phys., 163, 523-534, 1961.

Dunlop, D.J., Hysteresis properties of magnetite and their dependence on particle size: A test of pseudo-single domain remanence models, $J$. Geophys. Res., 91, 9569-9584, 1986.

Dunlop, D.J., Developments in rock magnetism, Rep. Prog. Phys., 53, 707792, 1990.

Dunlop, D.J., and M.-M. Bina, The coercive force spectrum of magnetite at high temperatures: evidence for the thermal activation below the blocking temperature, Geophys. J. R. Astron. Soc., 51, 121-147, 1977.

Fletcher, E., and W. O. Reilly, Contribution of $\mathrm{Fe}^{2+}$ jons to the magneto crystalline anisotropy constant $\mathrm{K}_{1}$ of $\mathrm{Fe}_{3 . x} \mathrm{Ti}_{x} \mathrm{O}_{4}(0<\mathrm{x}<0.1)$, J. Phys. $C$
Solid State Phys., 7, 171-178, 1974.

Halgedahl, S.L., Domain pattern observations in rock magnetism: progress and problems; Phys. Earth Planet. Inter., 46, 127-163, 1987.

Halgedahl, S.L., Magnetic domain patterns observed on synthetic Ti-rich titanomagnetite as a function of temperature and in states of thermoremanent magnetization, J. Geophys. Res., 96, 3943-3972, 1991.

Heider, F., and W. Williams, Note on temperature dependence of exchange constant in magnetile, Geophys. Res. Lett., 15, 184-187, 1988.

Heider, F., and V. Hoffmann, Magneto-opucal Kerr effect on magnetic crystals with externally applied fields. Earth Planet. Sci. Lett., 108, 131. 138, 1992.

Heider, F., D. J. Dunlop, and N. Sugiura, Magnetsc propertes of hydrothermally recrystallized magnetic crystals, Science, 236, 1287-1290, 1987.

Heider, F., D. J. Dunlop, and H.C. Soffel. Low-temperature and alternating field demagnetization of saturation remanence and thernoremanence in magnetite grains (0.037 $\mu \mathrm{m}$ to $5 \mathrm{~mm})$, J. Geophys. Res., 97, 9371-9381, 1992.

Hilzinger, H.R., The influence of planar defects on the coercive field of hard magnetic materials, Appl. Phys., 12, 253-260, 1977.

Hodych, J.P., Magnetostrictive control of coercive force in multidomain magnetite, Nature, 298, 542-544, 1982.

Hodych, J.P., Evidence for magnetostrictive control of intrinsic susceptibility and coercive force of multidomain magnetite in rocks, Phys. Earth Planet. Inter., 42, 184-194, 1986.

Hull, D., Introduction to Dislocations, Pergamon, New York, 1975.

Jakubovics, J.P., Comments on the definition of ferromagnetic domain wall widths, Philos. Mag. B, 38, 401-406, 1978.

Jakubovics, J.P., A.J. Lapworth, and T.W. Jolly, Electron microscope studies of ferromagnetic ordered structures, J. Appl. Phys., 49, 20022006, 1978.

Kittel, C., Physical theory of ferromagnelic domains, Rev. Mod. Phys., 2J, 541-583, 1949.

Klapel, G.D., and P.N. Shive, High-lemperature magnetostriction of magnetite, J. Geophys. Res..79, 2629-2633, 1974.

Landau, L., and L. Lifshitz, On the theory of the dispersion of magneuc permeability in ferromagnetic bodies, Phyz. Z., 8, 153-169, 1935.

Moon, T.S., Domain states in fine particle magnelite and titanomagnetite, J. Geophys. Res., 96, 9909-9923, 1991.

Moon, T.S., and R.T Merrill, The magnetic moments of non-uniformly magnetized grains, Phys. Earth Planet. Inter., 37, 186-194, 1984.

Moskowitz, B.M., High-temperature magnetostriction of magnetite and titanomagnetite, J. Geophys. Res.,98, 359-371 1993.

Moskowitz, B.M., and S.L. Halgedahl. Theoretical temperature and grain-size dependence of domain state in $\mathrm{x}=0.6$ titanomagnetite, $J$. Geophys. Res., 92, 10667-10682, 1987.

Moskowitz, B.M., S.L. Halgedahl, and C.A. Lawson, Magnetic domains on unpolished and polished surfaces of titanium-rich titanomagnetite, $J$. Geophys. Res., 93, 3372-3386, 1988.

Newell, A.J., D.J. Dunlop, and R.J. Enkin, Temperature dependence of critical sizes, wall widths and moments in two-domain magnetite grains, Phys. Earth Planet. Inter., 65, 165-176, 1990.

Pauthenet, P.R., Anımantation spontanee des fertutes, Ann. Phys., 7, 710-747, 1952.

Press, W.H., B.P. Flannery, S.A. Teukolsky, and W.T. Vetterling, Numerical Recipes in C: The Ant of Scientific Computing, Cambridge University Press, New York, 1988.

Smith, G.H, and R.T Merrill. Domain wall resonance and stability in magnetite, J. Geophys. Res.,88, 637-644, 1983.

Stacey, F.D., and K.N. Wise, Crystal dislocations and coercivity in finegrained magnetite, Aust. J. Phys., 20, 507-513, 1967.

Träuble, $H$., The influence of crystal defects on magnetization processes in ferromagnetic single crystals, in Magnetism and Metallurgy, pp. 622688, edited by A. E. Berkowitz and E. Kneller, Academic Press, San Diego, Calif., 1969.

Veyssière, P., J. Rabier, H. Garem, and J. Grilhé, Influence of temperature on dissociation of dislocations and plastic deformation in spinel oxıdes, Philos. Mag. A, 38, 61-79, 1978.

Worm, H.-U., M. Jackson, P. Kelso, and S.K. Banerjee, Thermal demagnetization of partial thermoremanent magnetization, J. Geophys. Res., 93, $12,196-12,204,1988$. 
$\mathrm{Xu}, \mathrm{S}$., and R.T. Merrill, Microstress and microcoercivity in multidomain grains, J. Geophys. Res., 94, 10,627-10,636, 1989.

$\mathrm{Xu}$, S., and R.T. Merrill, Microcoercivity, bulk coercivily, and saluration remanence in multidomain materials, J. Geophys. Res., 95, 7083-7090, $1990 a$.

Xu, S., and R.T. Merrill, Thermal variations of domain wall ulickness and number of domains in magnetic rectangular grains, J. Geophys. Res., 95, 21433-21,440, $1990 b$.
$\mathrm{Xu}, \mathrm{S}$, and R.T. Merrill, Stress, grain size, and magnetic stability of magnetite, J. Geophys. Res., 97, 4321-4329, 1992.

B.M. Moskowitz, Department of Geology and Geophysics, 310 Pillsbury Dr. S.E., University of Minnesota, Minneapolis, MN 55455.

(Received January 4, 1993;

revised June 7, 1993;

accepted June 22, 1993.) 